\title{
$-31686$
}

UCRL-ID-128000

\section{Chemical and Isotopic Data for Groundwater in Southern Nevada}

\author{
Timothy P. Rose \\ Jacqueline M. Kenneally \\ David K. Smith \\ M. Lee Davisson \\ G. Bryant Hudson \\ Jo Ann H. Rego
}

July 1997

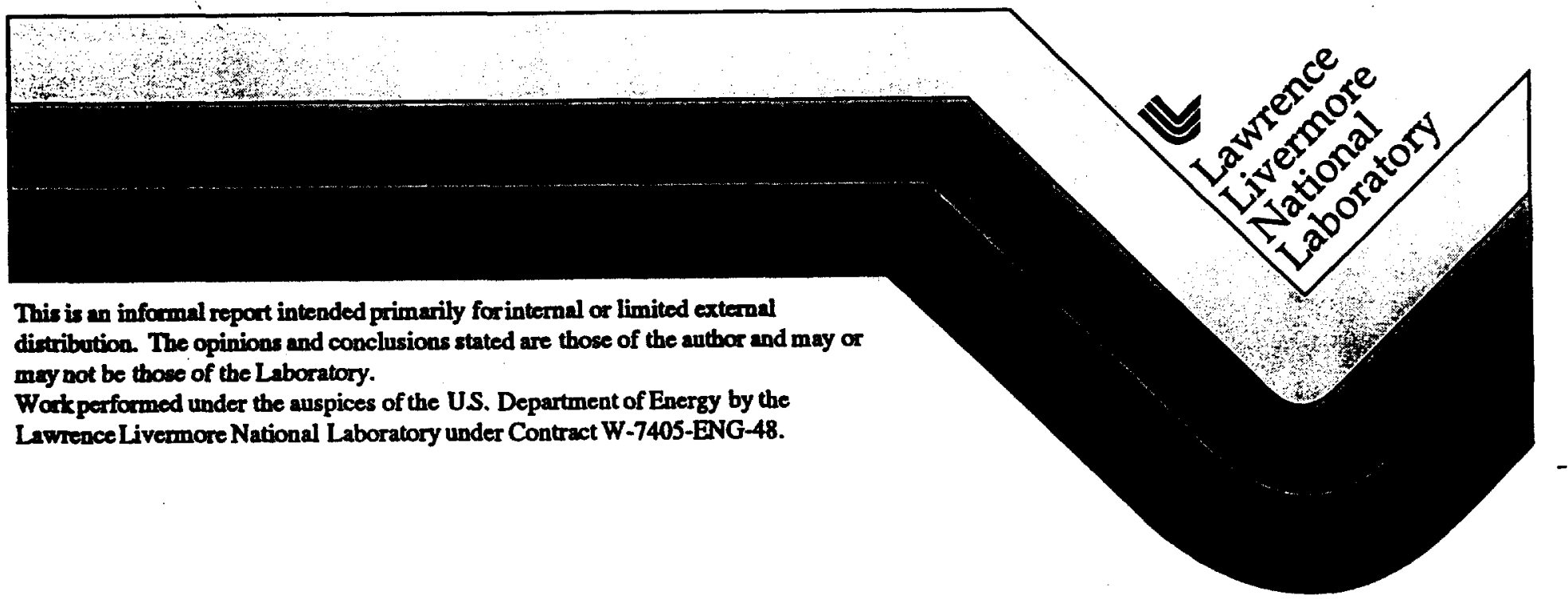




\section{DISCLAIMER}

This documeat was prepared as an account of work sponsored by an agency of the United States Government. Neither the United States Government nor the University of California nor any of their emplojees, makes any warranty, express or implied, or sesumes any legal liability or responsibility for the accuracy, completeness, or usefulaess of any information, apparatus, product, or process disclosed, or represents that its use would not infringe privately owned rights. Reference herein to any specinic commercial products, process, or service by trade name, trademark, manufacturer, or otherwise, does not necessarily comstitute or imply its endorsement, recommendation, or favoring by the United States Government or the University of Calffornia. The vews and opinions of authors expressed herein do not necessarily state or refiect those of the United States Government or the University of Calffornia, and shall not be used for advertising or product endorsemeat purposes.

This report has been reproduced directly from the best available cops.

Available to DOE and DOE contractors from the Orice of Scientific and Technical Information

P.O. Box 62, Oak Ridge, TN 37831

Prices available from (615) 576-8401, FTS 626-8401

Available to the public from the

National Technical Information Service

US. Department of Commerce

5285 Port Royal Rd.

Springfield, VA 22161 


\title{
CHEMICAL AND ISOTOPIC DATA FOR GROUNDWATER IN SOUTHERN NEVADA
}

\author{
Timothy P. Rose \\ Jacqueline $M$. Kenneally \\ David K. Smith \\ M. Lee Davisson \\ G. Bryant Hudson \\ Jo Ann H. Rego \\ Isotope Sciences Division \\ Lawrence Livermore National Laboratory \\ Livermore, CA 94550
}

July, 1997 


\section{EXECUTTVE SUMMARY}

This document presents a compilation of chemical and isotopic data for groundwater samples analyzed by Lawrence Livermore National Laboratory (LLNL) in support of the Hydrology and Radionuclide Migration Program (HRMP) and the Underground Test Area Program (UGTA) for the U.S. Department of Energy, Nevada Operations Office. Included are data for 107 samples collected from wells and springs located on and around the Nevada Test Site (NTS), within an area approximately bounded by latitudes $36^{\circ}$ to $38^{\circ} 15^{\prime} \mathrm{N}$ and longitudes $115^{\circ}$ to $117^{\circ} 15^{\prime} \mathrm{W}$. The samples were collected during the time period 1992 to early 1997 . The data represents one of the largest internally consistent geochemical data sets to be gathered for groundwater in southern Nevada. This database is available in electronic or hardcopy formats to interested parties upon request.

In addition to the LLNL data, we have included a table of selected isotopic data -summarized from a larger database compiled by GeoTrans, Inc. (1994). This data is included for comparative purposes as a means of placing the LLNL data in the context of other data for the same geographic region.

\section{INTRODUCTION}

The Isotope Sciences Division at Lawrence Livermore National Laboratory maintains a comprehensive database for chemical and isotopic analyses of groundwaters collected throughout southern Nevada. Although some of this data was previously reported in Davisson et al. (1994), a number of additional samples have been measured since that time. Details of more recent sampling efforts are given in Smith et al. $(1996,1997)$. Numerous requests for an updated version of this database have prompted the release of this document. An electronic version of this database (Microsoft Excel v. 5.0) is available upon request. Readers are encouraged to report errors to Tim Rose at the Isotope Sciences Division, L-231, Lawrence Livermore National Laboratory, Livermore, CA 94550; phone: (510) 422-6611; fax: (510) 422-3160; e-mail: rose23@llnl.gov.

Although this document does not include data interpretations, it precedes a manuscript in preparation that provides a detailed discussion of available stable isotope and ${ }^{14} \mathrm{C}$ data from southern Nevada (Davisson et al., 1997). Laboratory analytical procedures are described in the ISD/LLNL Standard Operating Procedures Manual for the UGTA Operable Unit (ISD, 1995), which is also available upon request. 


\section{LLNL DATA TABLES}

The LLNL data is separated into four tables arranged according to increasing sample latitude. The first two columns of each table are the same, showing sample numbers (between 1 and 107) followed by a "sample identification" that provides the location name and brief descriptive information. Wells from the NTS are identified relative to the NTS Area where each is located. When multiple samples were collected from different depth intervals using a bailer, the sample depth is indicated in meters below surface level (mbsl). When a particular location was sampled on more than one occasion, the sampling date (month/year) is indicated in parentheses along with the sample name.

Table 1 provides geographical sample locations and sample acquisition dates $(\mathrm{m} / \mathrm{d} / \mathrm{y})$. The geographical data includes both the latitude and longitude, as well as the township, range and section. Figure 1 is a map of southwestern Nevada showing sample locations. Numbered sample sites correspond to individual samples listed in Tables 1 through 4.

Table 2 describes field data and major ion chemistry. Field parameters include water temperature, electrical conductivity, dissolved oxygen (DO), and $\mathrm{pH}$. The total dissolved inorganic carbon (TDIC, reported in $\mathrm{mg} / \mathrm{L}$ as bicarbonate) was determined in the laboratory by acidifying the water sample under vacuum and cryogenically trapping the evolved $\mathrm{CO}_{2}$ gas (Davisson and Velsko, 1994). Other anion concentrations ( $\mathrm{F}, \mathrm{Cl}^{-}$and $\mathrm{SO}_{4}^{2-}$ ) were determined by ion chromatography. Major cation concentrations $\left(\mathrm{Na}^{+}, \mathrm{K}^{+}, \mathrm{Ca}^{2+}\right.$ and $\mathrm{Mg}^{2+}$ ) were measured by inductively-coupled plasma atomic emission spectroscopy.

Table 3 describes the trace element chemistry for 14 different elements, reported in units of $\mathrm{mg} / \mathrm{L}$. Li, $\mathrm{B}, \mathrm{Al}, \mathrm{Si}, \mathrm{Mn}, \mathrm{Fe}, \mathrm{Ni}, \mathrm{Sr}, \mathrm{Mo}$, and $\mathrm{Ba}$ were measured by inductivelycoupled plasma atomic emission spectroscopy whereas $\mathrm{As}, \mathrm{Se}, \mathrm{Pb}$, and $\mathrm{U}$ were measured by inductively-coupled plasma mass spectrometry.

Table 4 describes the results of isotopic measurements. Oxygen, hydrogen and carbon stable isotope ratios were measured on an isotope ratio mass spectrometer and are reported in the standard delta $(\delta)$ notation as per mil $(\%)$ deviations from SMOW (oxygen and hydrogen) or PDB (carbon) reference standards (cf. Criss, 1994). Carbon-14 and ${ }^{36} \mathrm{Cl}$ were measured on the accelerator mass spectrometer at Lawrence Livermore National Laboratory. The ${ }^{14} \mathrm{C}$ results are reported as percent modern carbon (pmc) relative to a NBS OX-1 standard that represents pre-nuclear testing atmospheric $\mathrm{CO}_{2}$ abundances (Stuiver and Polach, 1977). Carbon-14 apparent ages (in years) were calculated using a ${ }^{14} \mathrm{C}$ half life of 5730 years (Walker et al., 1989). The chlorine-36 results are reported as ${ }^{36} \mathrm{Cl} \mathrm{Cl}_{\text {TOTAL }}\left(x 10^{-13}\right)$ ratios measured on $\mathrm{AgCl}$ precipitated from water (Elmore et al., 1979). ${ }^{87} \mathrm{Sr} /{ }^{86} \mathrm{Sr}$ and ${ }^{234} \mathrm{U} /{ }^{238} \mathrm{U}$ isotope ratios were determined on pure elemental separates using thermal ionization mass spectrometry. Dissolved noble gas abundances were 
analyzed on a noble gas mass spectrometer. To determine the ${ }^{3} \mathrm{He} / \mathrm{He}$ ratio relative to air, the ${ }^{3} \mathrm{He} / \mathrm{He}$ values reported in Table 4 should be divided by $1.38 \times 10^{-6}$.

\section{GEOTRANS DATA COMPILATION}

Table 5 is a summary of selected isotopic data obtained from a database compiled by GeoTrans, Inc. (1994) for the U.S. Department of Energy, Nevada Operations Office. Our purpose in including it here is to provide a means of comparing the LLNL data set with other environmental isotope data from the same geographic area. The GeoTrans database was compiled from a variety of sources that include published literature as well as unpublished data from Lawrence Livermore National Laboratory, the U.S. Geological Survey, the Desert Research Institute, the U.S. Environmental Protection Agency, and the Reynolds Electrical Engineering Corporation (GeoTrans, 1994). Readers should refer to the $1994 \mathrm{GeoTrans}$ database for the appropriate references for specific data entries.

The format and contents of Table 5 differs from the GeoTrans compilation in several ways. Table 5 does not include every location listed in the GeoTrans data compilation. Data was included if both a $\delta^{18} \mathrm{O}$ and $\mathrm{a} \delta \mathrm{D}$ analysis was available for a particular site. The GeoTrans compilation uses a master ID number as the identifier for a particular location. We have not included this number here, although the data in Table 5 can be easily referenced to the GeoTrans compilation using the latitude and longitude data. The first column in Table 5 gives the sample name, and when possible, a brief geographic description. In a number of cases, the original data compilation did not include a sample name. We verified each location name by plotting the coordinates on a map, and cross referencing the coordinates with original references whenever possible.

The second column in Table 5 gives the township, range, and section coordinates for the sample. These coordinates were not included in the original compilation, but this information was readily determined during the location verification process. Columns 3 and 4 list the sample latitude and longitude. As with Tables 1-4, Table 5 is arranged according to increasing sample latitude. In most cases, the latitude and longitude reported in Table 5 match the coordinates given by GeoTrans, but when errors were found, a new set of coordinates were entered.

It should be noted that the LLNL data that appeared in the original GeoTrans compilation (samples collected before November, 1994) is not duplicated in Table 5. However, when a well was sampled by both LLNL and by another agency, all the data is included in Table 5 as an averaged value. The total number of analyses used to obtain the average is shown in parentheses following the data entry. Significant outliers were not included in this average. For example, in some cases two pairs of $\delta^{18} O$ and $\delta \mathrm{D}$ values 
were reported, but one set of analyses showed a marked isotopic enrichment, presumably due to evaporation. Under these circumstances, only the relatively unevaporated values are reported.

Slight discrepancies may exist between the latitude and longitude of samples that appear in both Table 1 and Table 5. In general, the coordinates given in Table 1 should be used for future reference to LLNL sample sites as these locations were carefully verified. Sampling dates in Table 5 are listed for locations that were sampled only once, but not for locations sampled on multiple occasions. In a few cases, sample dates were unavailable. Oxygen, hydrogen and carbon isotopic values are reported using the same conventions adopted for Table 4.

\section{ACKNOWLEDGMENTS}

We acknowledge the assistance of Rick Waddell and Chen Zhu of HSI GeoTrans, Inc. in providing us with a copy of their data compilation. We thank Greg Nimz for his efforts during the early stages of the project, and Jordan Clark for his laboratory assistance in measuring dissolved noble gas abundances. We also wish to acknowledge the Nevada Operations Office of the U.S. Department of Energy for HRMP and UGTA funding from FY91-FY97 to collect this data. This work was performed under the auspices of the U.S. Department of Energy by Lawrence Livermore National Laboratory under contract number W-7405-Eng-48. 


\section{REFERENCES}

Criss, R.E. (1994) Stable isotope distribution: Variations from temperature, organic and water-rock interactions. In: Handbook of Physical Constants, Sec. 1-20. Am. Geophys. Union, 14 p.

Davisson, M.L. and Velsko, C.A. (1994) Rapid extraction of dissolved inorganic carbon from small volumes of natural waters for ${ }^{14} \mathrm{C}$ determination by accelerator mass spectrometry. Lawrence Livermore National Laboratory Report UCRL-JC-119176, 22 p.

Davisson, M.L., Kenneally, J.M., Smith, D.K., Hudson, G.B., Nimz, G.J. and Rego, J.H. (1994) Preliminary report on the isotope hydrology investigations at the Nevada Test Site: Hydrologic Resources Management Program, FY 1992-1993. Lawrence Livermore National Laboratory Report UCRL-ID-116122, 82 p.

Davisson, M.L., Smith, D.K, Kenneally, J.M. and Rose, T.P. (1997) Isotope hydrology of southern Nevada groundwater. Manuscript in preparation.

Elmore, D., Fulton, B.R., Clover, M.R., Marsden, J.R., Gove, H.E., Naylor, H., Purser, K.H., Kilius, L.R., Beukens, R.P. and Litherland, A.E. (1979) Analysis of ${ }^{36} \mathrm{Cl}$ in environmental water samples using an electrostatic accelerator. Nature, 277: 22-25.

GeoTrans, Inc. (1994) A comprehensive chemistry database for groundwater and springs at the Nevada Test Site and vicinity. Unpublished report describing database prepared for the U.S. Department of Energy, November 22, 1994, 32 p.

Isotope Sciences Division (1995) Underground Test Area Remedial Investigation Feasibility Study, Standard Operating Procedures. Lawrence Livermore National Laboratory, unpublished report.

Smith, D.K., Esser, B.K., Kenneally, J.M., Nagle, R.J., Rego, J.H. and Royval, J.L. (1996) Hydrologic Resources Management Program, FY 1995 Progress Report. Lawrence Livermore National Laboratory Report UCRL-ID-123486, 36 p.

Smith, D.K., Kersting, A.B., Kenneally, J.M., Rego, J.H. and Thompson, J.L. (1997) Hydrologic Resources Management Program, FY 1996 Progress Report. Lawrence Livermore National Laboratory Report UCRL-ID-126886, 40 p.

Stuiver, M. and Polach, H. (1977) Reporting of ${ }^{14} \mathrm{C}$ data. Radiocarbon, 19: 355-363.

Walker, F.W., Parrington, J.R. and Feiner, F. (1989) Nuclides and Isotopes. General Electric Co. Nuclear Energy Operations, San Jose, California, 57 p. 
Figure 1. (overleaf) Map of southwestern Nevada showing locations of groundwater samples analyzed by Lawrence Livermore National Laboratory. Numbered sample sites correspond with individual samples listed in Tables 1 through 4. Base map is the U.S. Geological Survey topographic sheet for the state of Nevada. 


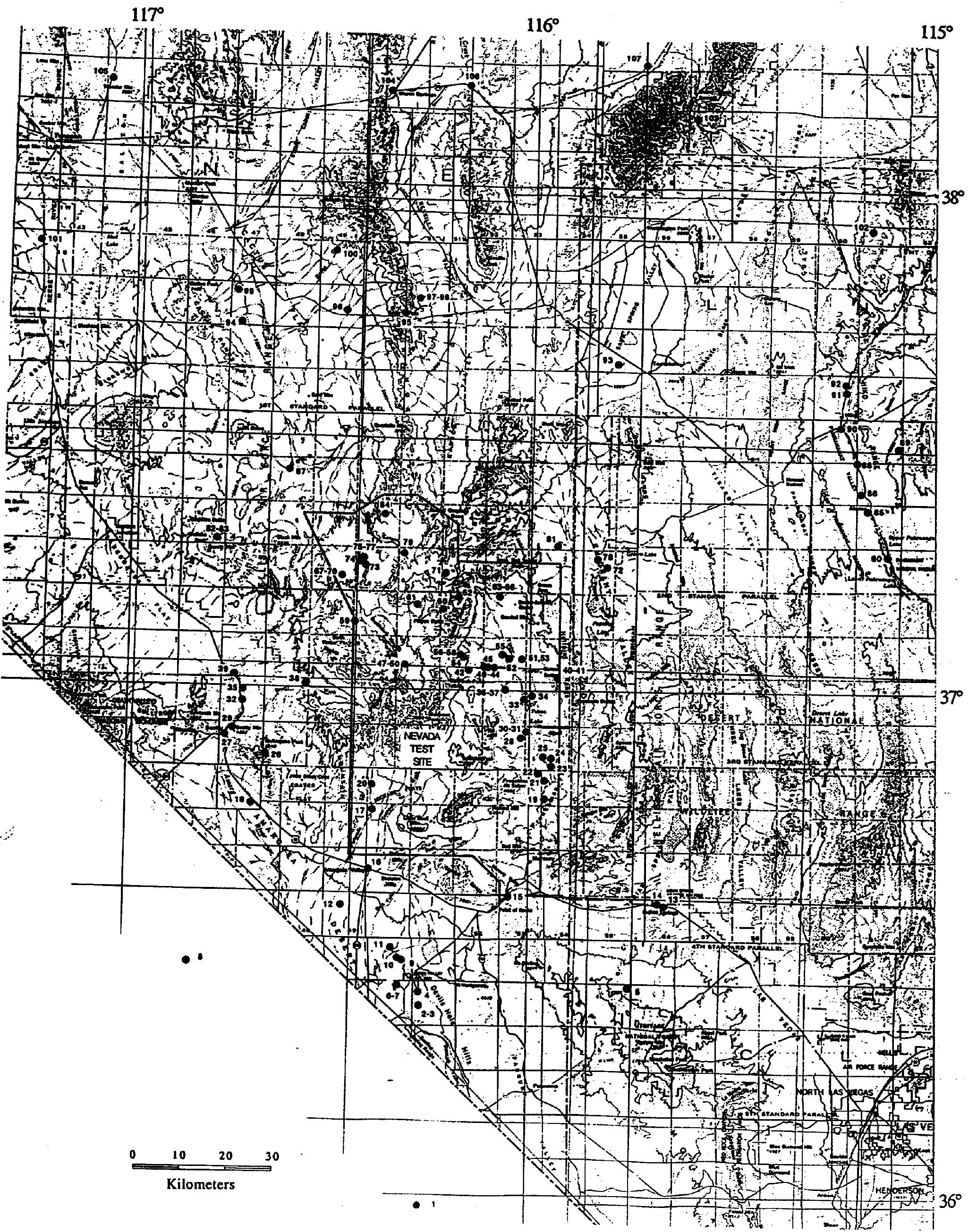


Table 1. Sample Location and Dato

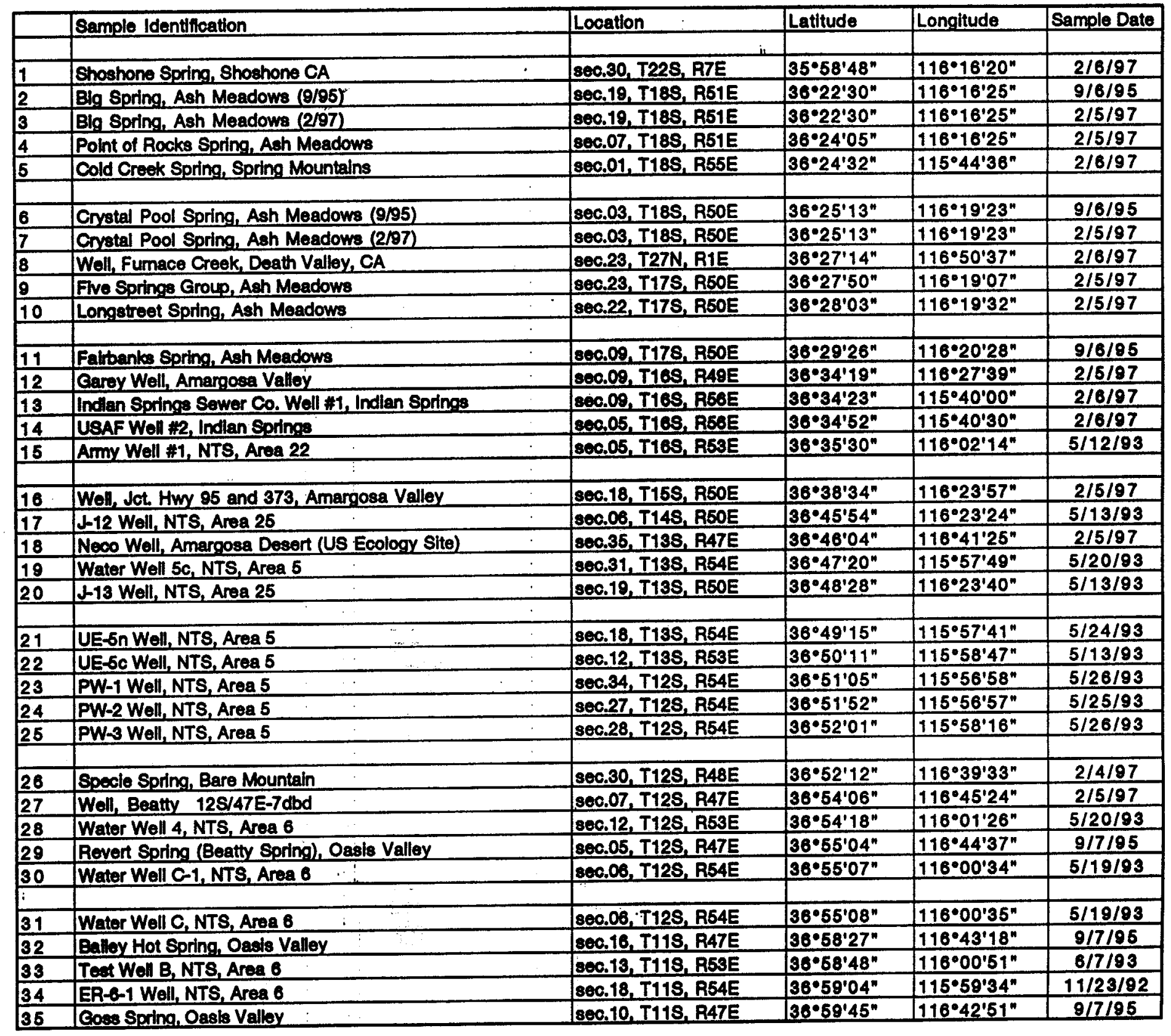

Coses Spring, Oasis Valley 


\begin{tabular}{|c|c|c|c|c|c|}
\hline & & & & & \\
\hline & Bample Identification & Location & Latitude & Longitude & Sample Date \\
\hline 37 & UE-1h Well, NTS, Area 1, 651 mbel & 800,09, T11S, R63E & $37^{\circ} 00^{\prime} 05^{\prime \prime}$ & $116^{\circ} 04^{\prime} 03^{\prime \prime}$ & $5 / 26 / 93$ \\
\hline 38 & Coffers Ranch, Windmill, Oasts Valley & $806,01, T 11 S, R 48 E$ & $37^{\circ} 00^{\prime} 16^{\prime \prime}$ & $116^{\circ} 33^{\prime} 26^{\prime \prime}$ & $2 / 4 / 97$ \\
\hline 39 & Unnamed Spring, Oagis Valloy & $80 C .32, T 10$, RA7E & $37^{\circ} 01^{\prime 1} 11^{\prime \prime}$ & $116^{\circ} 44^{\prime} 00^{\prime \prime}$ & $2 / 4 / 97$ \\
\hline 40 & ER-3-1 Well, NTS, Area 3, (9/95) & $800.35, T 108$, R54E & $37^{\circ} 01^{\prime} 33^{\prime \prime}$ & $115^{\circ} 56^{\prime} 13^{\prime \prime}$ & 9/11/95 \\
\hline & & log 35 T10S R54F & $37^{\circ} 01^{\prime} 33^{\prime \prime}$ & $115^{\circ} 56^{\prime} 13^{n}$ & $10 / 16 / 96$ \\
\hline$\frac{41}{42}$ & $\begin{array}{l}\text { ER-3-1 Well, NTS, Area 3, (10/96) } \\
\text { UE-16f Well, NTS, Area } 18\end{array}$ & $\begin{array}{l}\text { 80c.35, T10S, R54E } \\
80 c .27, T 10 S, \text { A52E }\end{array}$ & $37^{\circ} 02^{\prime} 09^{\prime \prime}$ & $116^{\circ} 09^{\prime} 25^{\prime \prime}$ & $\frac{10 / 18 / 96}{7 / 12 / 93}$ \\
\hline$\frac{42}{43}$ & $\begin{array}{l}\text { UE-16f Well, NTS, Area } 18 \\
\text { UE-1c Well, NTS, Area } 1,411 \mathrm{mbs}\end{array}$ & sec.20,T10S, R53E & $37^{\circ} 02^{\prime} 63^{\prime \prime}$ & $116^{\circ} 05^{\prime} 52^{\prime \prime}$ & $9 / 2 / 92$ \\
\hline$\frac{50}{44}$ & UE-10 Well, NTS, Area 1, $499 \mathrm{mbsi}$ & Soc.20, T10S, R53E & $37^{\circ} 02^{\prime} 53^{\prime \prime}$ & $116^{\circ} 05^{\prime} 52^{\prime \prime}$ & $9 / 2 / 92$ \\
\hline 45 & UE-16 Well, NTS, Area 1 & $800.19, T 108$, R63E & $37^{\circ} 02^{\prime} 54^{\prime \prime}$ & $116^{\circ} 06^{\prime} 42^{\prime \prime}$ & $8 / 31 / 92$ \\
\hline & & & & & \\
\hline 46 & UE-1a Well, NTS, Area 1 & SOC.19, T10S, R53E & $37^{\circ} 02^{\prime} 54^{\prime \prime}$ & $116^{\circ} 07^{\prime} 06^{\prime \prime}$ & 9/1/92 \\
\hline 47 & ER-30-1 Well, String \#1, NTS, Aroa 30 (1/95) & $80 C .20, T 10$ S, R51E & $37^{\circ} 03^{\prime} 01^{\prime \prime}$ & $116^{\circ} 18^{\prime} 58^{\prime \prime}$ & $1 / 31 / 95$ \\
\hline 48 & ER-30-1 Well, String \#2, NTS, Area 30 (2/95) & 80c.20, T10S, R51E & $37^{\circ} 03^{\prime} 01^{\prime \prime}$ & $116^{\circ} 18^{\prime} 58^{\prime \prime}$ & $2 / 1 / 95$ \\
\hline 49 & ER-30-1 Well, String \#1, NTS, Area 30 (6/96) & $800.20, T 10 S, \mathrm{R} 51 \mathrm{E}$ & $37^{\circ} 03^{\prime} 01^{\prime \prime}$ & $116^{\circ} 18^{\prime} 58^{n}$ & $6 / 10 / 96$ \\
\hline 50 & ER-30-1 Well, String \#2, NTS, Area 30 (6/98) & $800.20, T 105$, R51E & $37^{\circ} 03^{\prime} 01^{\prime \prime}$ & $116^{\circ} 18^{\prime} 58^{\prime \prime}$ & $6 / 10 / 96$ \\
\hline & & & & & \\
\hline 51 & U3-an $\# 5$ Well, NTS, Area 3 & 80C.24, T10S, R53E & $37^{\circ} 03^{\prime} 34^{\prime \prime}$ & $116^{\circ} 01^{\prime} 20^{\prime \prime}$ & $1 / 29 / 97$ \\
\hline 52 & UE-19 Woll, NTS, Aren 1 & Sec.15, T10S, R53E & $37^{\circ} 03^{\prime} 37^{\prime \prime}$ & $116^{\circ} 03^{\prime} 30^{\prime \prime}$ & $7 / 10 / 92$ \\
\hline 53 & US-en PS \#2 Well, NTS, Area 3 & Bec.13, T103, R59E & $37^{\circ} 03^{\prime} 38^{\prime \prime}$ & $116^{\circ} 01^{\prime} 18^{\prime \prime}$ & $1 / 22 / 97$ \\
\hline 54 & UE-16d Well, NTS, Area 16 & $800.15, T 10 S, R 52 E$ & $37^{\circ 0} 04^{\prime} 06^{\prime \prime}$ & $116^{\circ} 09^{\prime} 56^{\prime \prime}$ & $6 / 2 / 93$ \\
\hline 55 & Test Well D, NTS, Area 4 & 806.16, T10S, R53E & $37^{\circ} 04^{\prime} 18^{\prime \prime}$ & $116^{\circ} 04^{\prime} 45^{\prime \prime}$ & $6 / 8 / 93$ \\
\hline & TIE-47a Wall NTS Ares $17.253 \mathrm{mbgl}$ & SEC.15, T10S, R52E & $37^{\circ} 04^{\prime} 19^{\prime \prime}$ & $116^{\circ} 10^{\prime} 17^{\prime \prime}$ & $6 / 9 / 93$ \\
\hline$\frac{56}{57}$ & $\begin{array}{l}\text { UE-17 a Well, NTS, Area 1, } 253 \text { moi } \\
\text { UE-17a Well, NTS, Area 17, } 302 \mathrm{mbsl}\end{array}$ & $\begin{array}{l}80 C .15, T 10 S, R 52 E \\
80 C .15, T 10 S, R 52 E\end{array}$ & $37^{\circ} 04^{\prime} 19^{\prime \prime}$ & $116^{\circ} 10^{\prime} 17^{\prime \prime}$ & $6 / 9 / 93$ \\
\hline 58 & UE-17a Well, NTS, Area 17, 331 mbal & $800.16, T 10 S, R 52 E$ & $37^{\circ} 04^{\prime} 19^{n}$ & $116^{\circ} 10^{\prime} 17^{n}$ & $8 / 9 / 93$ \\
\hline 59 & UE-18r Well, NTS, Area 18 & $800.24, T 98$, R49E & $37 \cdot 08^{\prime} 05^{\prime \prime}$ & $116^{\circ} 26^{\prime} 41^{\prime \prime}$ & $8 / 11 / 92$ \\
\hline 60 & Teot Well 1, NTS, Area 17 & 80C. 18, TOS, R52E & $37^{\circ} 09^{\prime} 29^{\prime \prime}$ & $116^{\circ} 13^{\prime} 23^{\prime \prime}$ & $8 / 13 / 92$ \\
\hline & & & & & \\
\hline 61 & Water Well 8, NTS, Area 18 & $800.09, \mathrm{~T} 9 \mathrm{~S}, \mathrm{R} 51 \mathrm{E}$ & $37^{\circ} 09^{\prime} 56^{\prime \prime}$ & $116^{\circ} 17^{\prime} 21^{\prime \prime}$ & $6 / 2 / 93$ \\
\hline 62 & ER-12-1 Well, NTS, Area 12 & $800.04, T 95$, R62E & $37^{\circ} 11^{\prime} 06^{\prime \prime}$ & $116^{\circ} 11^{\prime} 03^{\prime \prime}$ & $1 / 6 / 93$ \\
\hline 63 & UE-10] Well, Composite, NTS, Area B (10/93) & 800.04, T9S, R53E & $37^{\circ 11} 1^{\circ} 08^{\prime \prime}$ & $116^{\circ} 04^{\prime} 53^{\prime \prime}$ & $10 / 3 / 93$ \\
\hline 64 & UE-10i Well, Zone 1, NTS, Area 8 (3/97) & SOC.04, T9S, R53E & $37^{\circ} 11^{\prime} 08^{\prime \prime}$ & $116^{\circ} 04^{\prime} 53^{\prime \prime}$ & $3 / 17 / 97$ \\
\hline 65 & UE-10) Well, Zone 2, NTS, Area 8 (3/97) & $800.04, T 9 S$, R63E & $37^{\circ} 11^{\prime} 08^{\prime \prime}$ & $110^{\circ} 04^{\prime} 53^{n}$ & $3 / 20 / 97$ \\
\hline & & & & & \\
\hline 66 & UE-10) Well, Zone 3, NTS, Area 8 (3/97) & S806.04, T9S, R53E & $37^{\circ} 11^{\prime} 08^{\prime \prime}$ & $1160^{\circ} 04^{\prime} 53^{\prime \prime}$ & $3 / 24 / 97$ \\
\hline 67 & ER-20-5 \#3 Well, NTS, Area 20 (7/96) & BOC.26, T8S, R49E & $37^{-13} 13^{\prime \prime}$ & $116^{\circ} 28^{\prime} 39^{\prime \prime}$ & $7 / 31 / 08$ \\
\hline 68 & ER-20-5 \# Well, NTS, Area 20 (4/97) & $800.26, T 8 S, R 49 E$ & $37^{\bullet} 13^{\prime} 13^{n}$ & $116^{\circ} 28^{\prime} 39^{n}$ & $4 / 22 / 97$ \\
\hline 69 & ER-20-5 \#1 Well, NTS, Area 20 (6/96) & 8ec.28, T8S, R49E & $37^{\circ} 13^{\prime} 14^{\prime \prime}$ & $116^{\circ} 28^{\prime} 39^{\prime \prime}$ & $8 / 3 / 96$ \\
\hline 70 & ER-20-5 \#1 Well, NTS, Area 20 (4/97) & 806.26, T88, R49E & $37^{\bullet} 13^{\prime} 14^{n}$ & $116^{\circ} 28^{\prime} 39^{\prime \prime}$ & $4 / 22 / 97$ \\
\hline
\end{tabular}


Table 1. Sample Location and Date

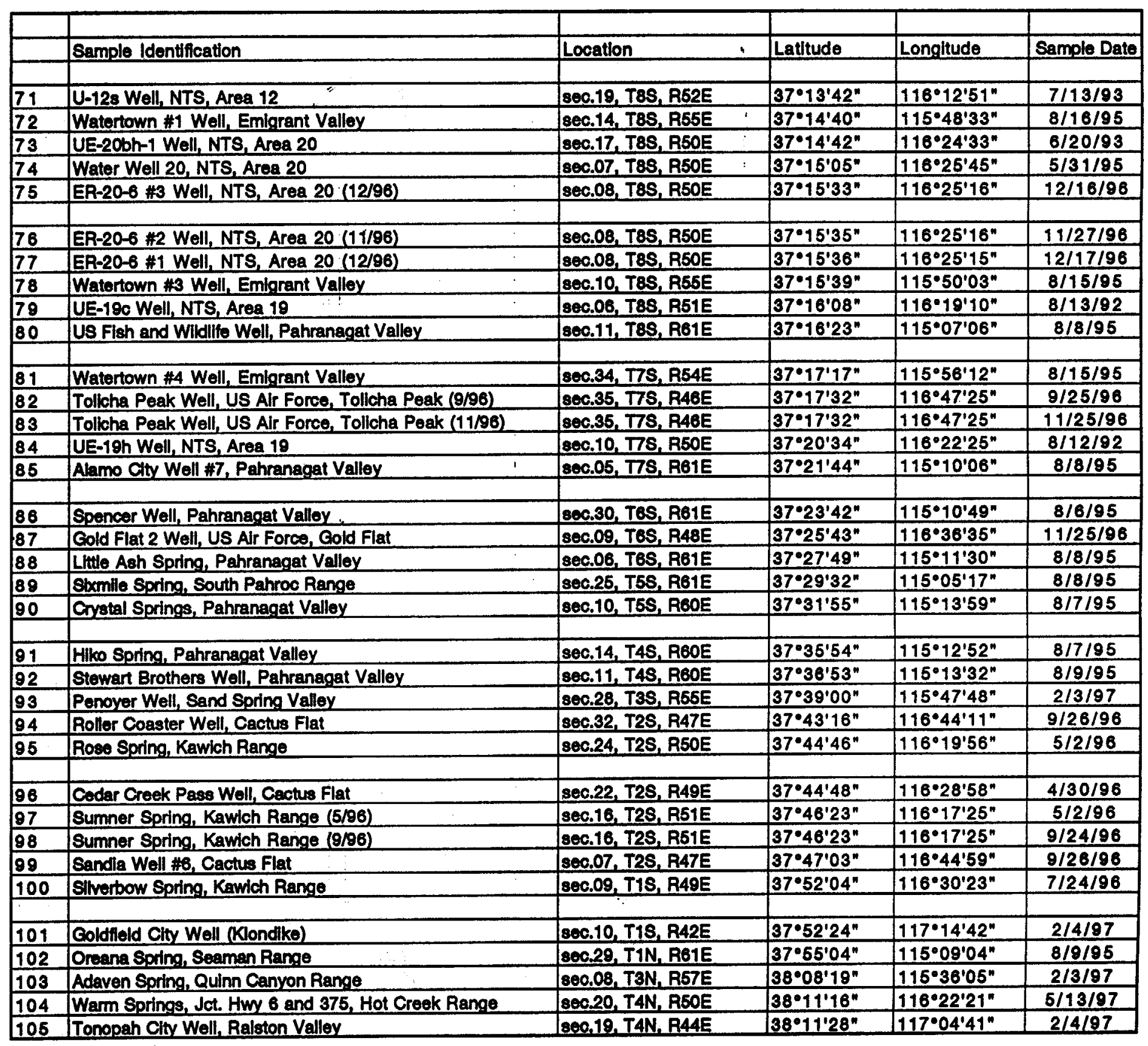


Table 1. Sample Location and Dato

\begin{tabular}{|l|l|l|l|c|c|}
\hline & & & \\
\hline & Sample Identification & Location & Latliude & Longltude & Sample Date \\
\hline & & & & & \\
\hline 106 & Twin Springs Ranch Well, Pancek6 Range & 806.13, T4N, R51E & $38^{\circ} 12^{\prime} 13^{\prime \prime}$ & $118^{\circ} 10^{\prime} 29^{\prime \prime}$ & $2 / 3 / 97$ \\
\hline 107 & Sharp Ranch Well (Nyala), Railióad Valley & $800.31, T 5 N, R 56 E$ & $38^{\circ} 14^{\prime} 56^{\prime \prime}$ & $115^{\circ} 43^{\prime} 40^{\prime \prime}$ & $2 / 3 / 97$ \\
\hline
\end{tabular}


Table 2. Flold data and major lon chemiotry

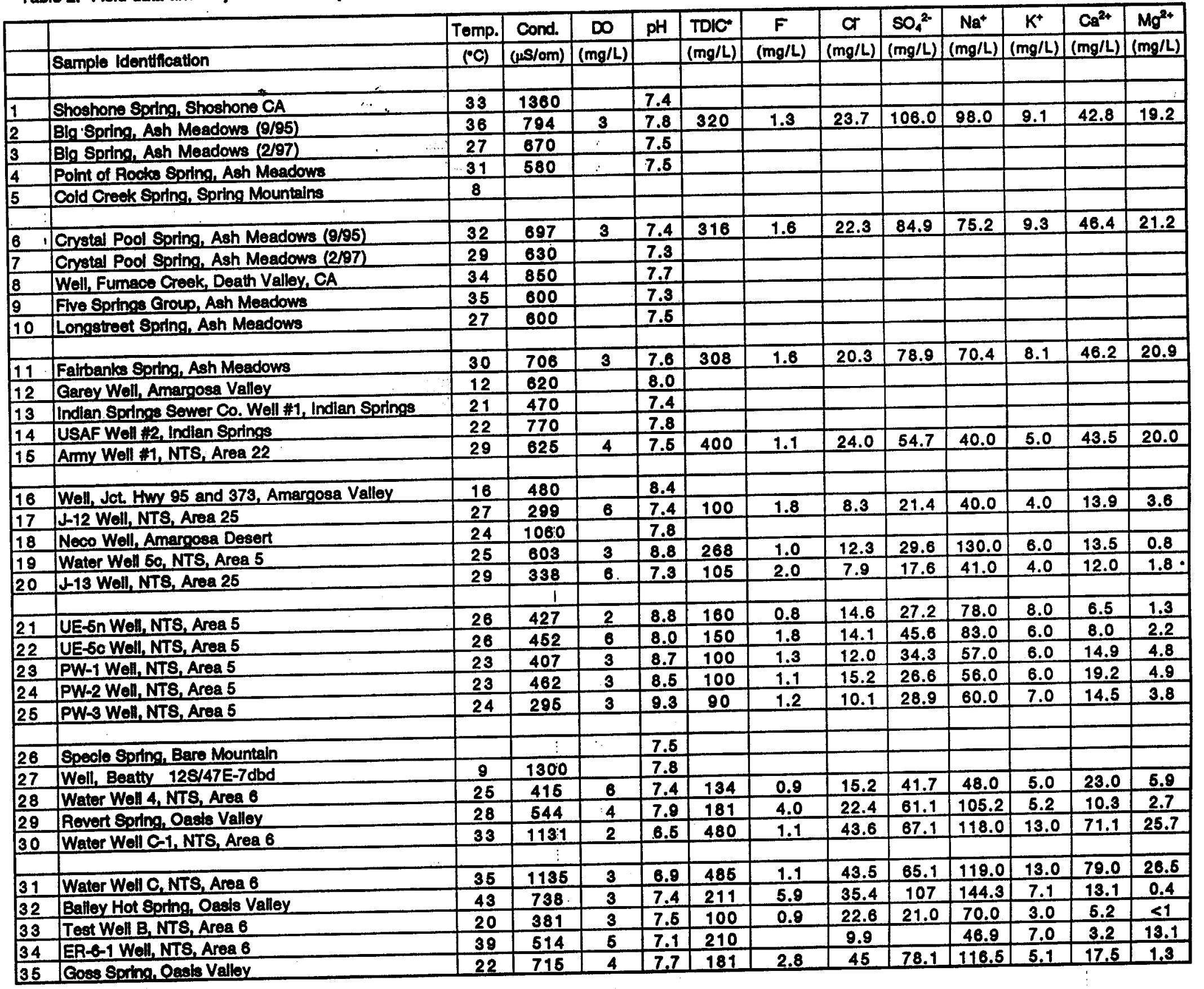


Table 2. Field data and mejor lon chemiery

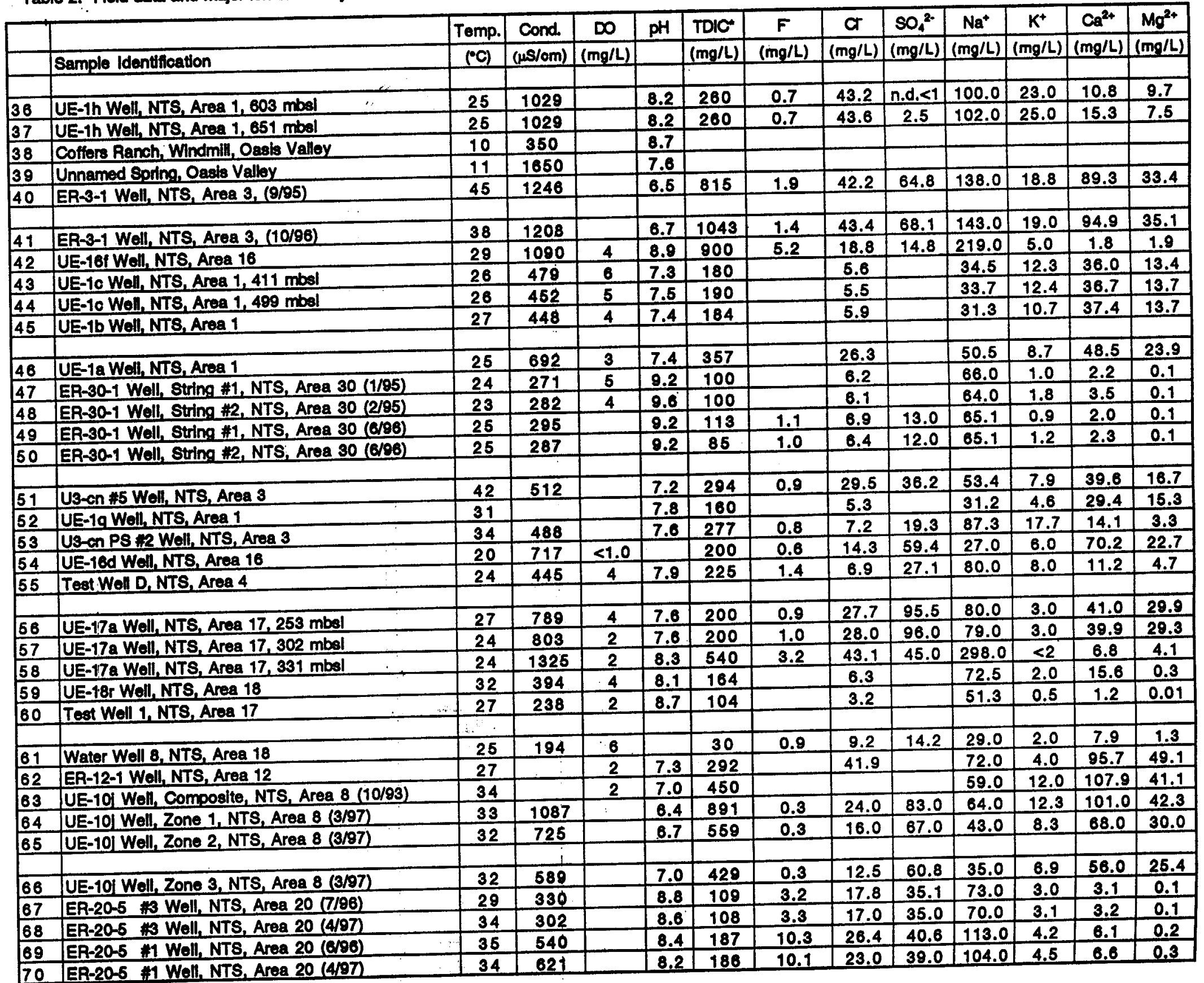


Table 2. Fleld data and major lon chemiatry

\begin{tabular}{|c|c|c|c|c|c|c|c|c|c|c|c|c|c|}
\hline & & Temp. & Cond. & D & pH & TDIC & $\mathbf{F}$ & $a$ & $\mathrm{SO}_{4}{ }^{2-}$ & $\mathrm{Na}^{+}$ & $\mathrm{K}^{+}$ & $\mathrm{Ca}^{2+}$ & $\mathbf{M g}^{2+}$ \\
\hline & Sample Identfication & $(\cdot 0)$ & $(\mu \mathrm{S} / \mathrm{om})$ & $(\mathrm{mg} / \mathrm{L})$ & & $(\mathrm{mg} / \mathrm{L})$ & $(\mathrm{mg} / \mathrm{L})$ & $(\mathrm{mg} / \mathrm{L})$ & $(\mathrm{mg} / \mathrm{L})$ & $(\mathrm{mg} / \mathrm{L})$ & $(\mathrm{mg} / \mathrm{L})$ & $(\mathrm{mg} / \mathrm{L})$ & $(\mathrm{mg} / \mathrm{L})$ \\
\hline & & & & & & & & & & & & & \\
\hline 71 & U-128 Well, NTS, Area 12 & 26 & 632 & 7 & 10.5 & 158 & n.d. $\leq 1$ & 14.0 & n.d. $<1$ & 24.0 & 5.0 & 35.6 & 0.1 \\
\hline 72 & Watortown *1 Well, Emigrant Valley & 24 & 330 & 2 & 7.7 & 167 & 2.3 & 6.9 & 19.6 & 89.3 & 8.2 & 3.8 & 1.1 \\
\hline 73 & UE-20bh-1 Well, NTS, Area 20 & 26 & 186 & 12 & 8.3 & 60 & n.d. $<1$ & 3.9 & n.d. $<1$ & 38.0 & $<2$ & 0.9 & n.d. $<1$ \\
\hline 74 & Water Well 20, NTS, Area 20 & 34 & 308 & & 8.2 & 92 & & 12.0 & & 59.0 & 2.1 & 6.2 & 0.3 \\
\hline \multirow[t]{2}{*}{75} & ER-20-6 \#3 Woll, NTS, Area 20 (12/96) & 28 & 310 & & 8.4 & 109 & 2.5 & 13.8 & 31.8 & 56.0 & 3.6 & 10.1 & 0.8 \\
\hline & & & & & & & & & & & & & \\
\hline 76 & ER-20-6 \#2 Well, NTS, Area 20 (11/96) & 26 & 308 & & 8.2 & 112 & 3.8 & 11.6 & 31.5 & 61.1 & 3.1 & 8.3 & 0.7 \\
\hline 77 & ER-20-6 \#1 Well, NTS, Area 20 (12/96) & 21 & 302 & & 8.1 & 103 & 2.9 & 12.3 & 32.3 & 60.6 & 2.2 & 7.1 & 0.6 \\
\hline 78 & Watertown $\# 3$ Well, Emigrant Valley & 23 & 42.5 & 3 & 7.9 & 223 & 1.0 & 9.5 & 33.1 & 82.0 & 7.5 & 24.4 & 5.7 \\
\hline 79 & UE-190 Woll, NTS, Area 19 & 37 & 174 & 6 & 7.7 & 37 & & 3.1 & & 35.8 & 0.5 & 1.4 & 0.01 \\
\hline \multirow[t]{2}{*}{80} & US Fish and Wildilite Well, Pahranagat Valley & 14 & 1297 & 2 & 7.8 & 628 & 1.6 & 83.0 & 192.0 & 130.3 & 19.3 & 79.5 & 53.7 \\
\hline & Watertown \#4 Well, Emiarant Valley & 25 & 1100 & 0.3 & 6.9 & 631 & 1.3 & 12.6 & 68.1 & 83.0 & 24.8 & 83.1 & \\
\hline 82 & Tollcha Peak Woll, Tollcha Peak (9/96) & $\frac{21}{31}$ & 407 & & 8.0 & & 0.9 & 26.0 & 39.1 & 57.8 & 4.2 & 24.4 & $\frac{24.4}{1.1}$ \\
\hline 83 & Tollcha Peak Well, Tollicha Peak (11/96) & 30 & 383 & & 7.9 & & & & & & & & \\
\hline 84 & UE-19h Well, NTS, Area 19 & 28 & 415 & 2 & 8.3 & 147 & & 8.5 & & 63.8 & 4.0 & 14.9 & 1.5 \\
\hline \multirow[t]{2}{*}{85} & Alamo Cty Well \#7, Pahranagat Valloy & 19 & 1032 & 4 & 7.6 & 454 & 1.3 & 54.6 & 188.0 & 96.3 & 13.7 & 61.4 & 56.0 \\
\hline & & & & & & & & & & & & & \\
\hline 86 & Spencer Well, Pahranagat Valloy & 19 & 1070 & 1 & 7.7 & 466 & 1.6 & 45.9 & 158.0 & 119.4 & 14.5 & 53.8 & 44.0 \\
\hline 87 & Gold Flat 2 Well, US Air Force, Gold Flat & 18 & 222 & & 8.0 & & 1.3 & 6.0 & 0.4 & & & & \\
\hline 88 & Little Ash Spring, Pahranagat Valloy & 37 & 468 & 2 & 7.4 & 250 & 0.8 & 8.5 & 35.0 & 29.8 & 7.3 & 45.3 & 15.4 \\
\hline 89 & Skxmile Spring, South Pahroc Range & 22 & 779 & 5 & 7.8 & 207 & 0.1 & 3.2 & 11.7 & 18.9 & 1.3 & 45.2 & 10.6 \\
\hline \multirow[t]{2}{*}{90} & Crystal Springs, Pahranagat Valley & 28 & 452 & 2 & 7.7 & 255 & 0.4 & 9.6 & 34.7 & 23.8 & 4.8 & 44.2 & 22.6 \\
\hline & Hilm ondme Po & & & & 7.7 & 273 & 0.5 & 46.3 & 38.1 & 25.6 & 6.6 & 46.4 & 23.3 \\
\hline$\frac{91}{92}$ & $\begin{array}{l}\text { Hilko Spring, Pahranagat Valley } \\
\text { Stewart Brothers Well, Pahranagat Valley }\end{array}$ & 27 & $\frac{542}{1582}$ & $\frac{2}{11}$ & $\frac{7.7}{7.8}$ & 361 & nd $<0.8$ & 13.9 & 438.0 & $\frac{20.0}{181.2}$ & 0.0 & $\frac{46.4}{90.0}$ & $\frac{23.3}{57.3}$ \\
\hline 93 & $\begin{array}{l}\text { Penoyer Well, Sand Spring Valloy } \\
\end{array}$ & 13 & 450 & & 7.3 & 181 & & & & & & & \\
\hline 94 & Roller Coaster Well, Cactus Fiat & 26 & 513 & & 7.8 & 120 & 0.9 & 38.0 & 34.0 & 65.2 & 8.0 & 21.3 & 1.6 \\
\hline \multirow[t]{2}{*}{95} & Rose Sping, Kawich Range & 18 & 688 & 5 & 7.4 & 354 & 0.3 & 23.0 & 49.5 & 44.0 & 1.9 & 82.4 & 11.2 \\
\hline & & & & & & & & & & & & & \\
\hline 96 & Codar Croek Pass Well, Cactus Flat & 28 & 288 & 6 & 7.7 & 105 & 0.5 & 15.2 & 25.0 & 34.7 & 9.4 & 20.7 & 0.5 \\
\hline 97 & Sumner Spring, Kawich Range (5/96) & 15 & 530 & 5 & 7.8 & 243 & 0.3 & 23.6 & 50.1 & 41.0 & 2.7 & 62.7 & 8.7 \\
\hline 98 & Sumner Spring, Kawlch Range (9/96) & & 551 & & 7.7 & & 0.4 & 22.8 & 50.5 & 43.1 & 2.1 & 65.8 & 9.0 \\
\hline 99 & Sandla Well :B, Cactus Flat & 23 & 624 & & 9.1 & 173 & 1.6 & 26.6 & 44.3 & 103.0 & 5.6 & 2.1 & $<0.01$ \\
\hline \multirow[t]{2}{*}{100} & Silverbow Spring, Kawich Range & 24 & 677 & 4 & .7 .1 & 270 & 0.4 & 22.6 & 43.3 & 47.0 & 2.3 & 49.1 & 9.1 \\
\hline & & & & & & & & & & & & & \\
\hline 101 & Goldifield City Well (Klondike) & 18 & 470 & & 8.2 & 173 & & & & & & & \\
\hline 102 & Oreana Spring, Soaman Range & 20 & 678 & & 7.7 & 392 & 0.2 & 17.5 & 30.0 & 25.5 & 1.4 & 97.5 & 12.2 \\
\hline 103 & Adaven Spring, Quinn Canyon Range & 10 & 500 & & 7.1 & 368 & & & & & & & \\
\hline 104 & Warm Spings, Hot Creok Range & 58 & 1322 & & 0.6 & & & & & & & & \\
\hline 105 & Tonopah City Well, Ralston Valley & 12 & 290 & & 7,4 & 195 & & & & & & & \\
\hline
\end{tabular}


Table 2. Fild data and major lon chamlety

\begin{tabular}{|c|c|c|c|c|c|c|c|c|c|c|c|c|c|}
\hline & & Temp. & Cond. & D & pH & TDIC: & $\mathbf{F}$ & a & $\mathrm{SO}_{4}{ }^{2-}$ & $\mathrm{Na}^{+}$ & $\mathbf{K}^{+}$ & $\mathrm{Ca}^{2+}$ & $\mathrm{Mg}^{2+}$ \\
\hline & Sample Identlification & (C) & (us/om) & $(\mathrm{mg} / \mathrm{L})$ & & $(\mathrm{mg} / \mathrm{L})$ & $(\mathrm{mg} / \mathrm{L})$ & $(\mathrm{mg} / \mathrm{h})$ & $(\mathrm{mg} / \mathrm{L})$ & $(\mathrm{mg} / \mathrm{h})$ & $(\mathrm{mg} / \mathrm{L})$ & $(\mathrm{mg} / \mathrm{L})$ & $(m g / L)$ \\
\hline & & & & & & & & & & & & & \\
\hline 108 & Twin Springs Ranch Well, Pancake Range & 12 & 570 & & 7.6 & 401 & & & & & & & \\
\hline 107 & Sharp Ranch Well (Nyala), Rallroad Valloy & 11 & 1200 & & 7.2 & 276 & & & & & & & \\
\hline & & & & & & & & & & & & & \\
\hline “TDII & - - Total Dlesolved Inorganic Carbon as HCO & & & & & & & & & & & & \\
\hline
\end{tabular}




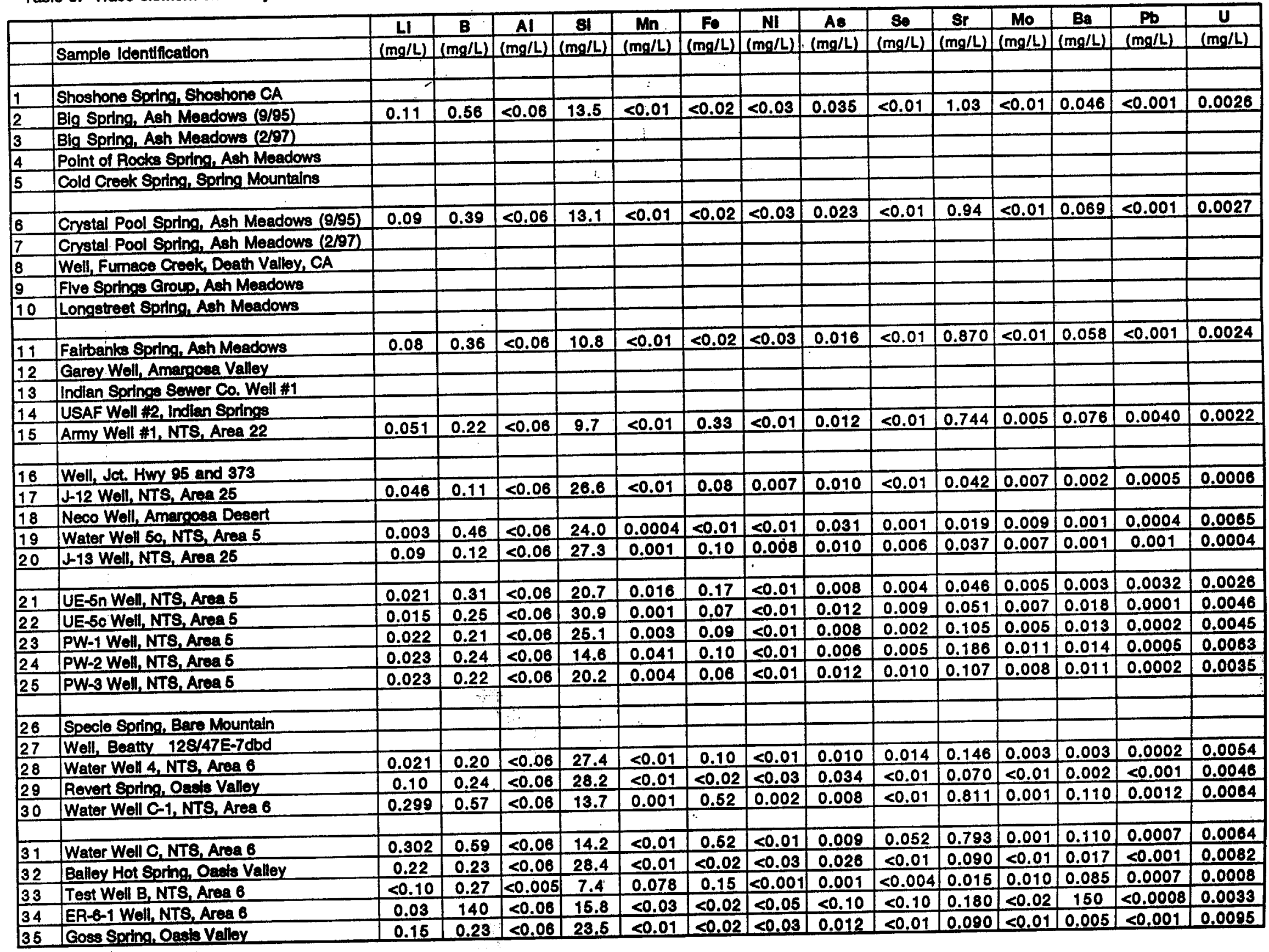




\begin{tabular}{|c|c|c|c|c|c|c|c|c|c|c|c|c|c|c|c|}
\hline & & 니 & $\mathbf{B}$ & Al & 81 & Mn & Fe & NI & As & Se & $8 \mathbf{s}$ & Mo & $\mathrm{Ba}$ & $P_{b}$ & $\mathbf{u}$ \\
\hline & Sample Identification & $(\mathrm{mg} / \mathrm{L})$ & $(\mathrm{mg} / \mathrm{l})$ & $(\mathrm{mg} / \mathrm{L})$ & $(\mathrm{mg} / \mathrm{L})$ & $(\mathrm{mg} / \mathrm{L})$ & $(m g / L)$ & $(\mathrm{mg} / \mathrm{L})$ & h $(\mathrm{mg} / \mathrm{L})$ & $(\mathrm{mg} / \mathrm{L})$ & $(\mathrm{mg} / \mathrm{L})$ & $(\mathrm{mg} / \mathrm{L})$ & $(\mathrm{mg} / \mathrm{l})$ & $(\mathrm{mg} / \mathrm{L})$ & $(\mathrm{mg} / \mathrm{L})$ \\
\hline & & & & & & & & & & & & & & & \\
\hline 36 & UE-1h Well, NTS, Area 1, 603 mbsl & $<0.1 .0^{2}$ & 0.26 & $<0.005$ & 4.5 & 0.041 & $<0.02$ & $<0.001$ & 0.003 & $<0.004$ & 0.169 & 0.066 & 0.055 & 0.0004 & $\leq 0.0004$ \\
\hline 37 & UE-1h Well, NTS, Area 1, $651 \mathrm{mbsl}$ & 0.083 & 0.17 & 0.04 & 5.5 & 0.024 & 0.12 & 0.010 & 0.006 & 0.004 & 0.200 & 0.061 & 0.064 & 0.0004 & 0.0001 \\
\hline 38 & Coffers Ranch, Windmill, Oasis Valloy & & & & & & & & & & & & & & \\
\hline 39 & Unnamed Spring, Oasts Valley & & & & & & & & & & & & & & \\
\hline \multirow[t]{2}{*}{40} & ER-3-1 Woll, NTS, Area 3, (9/95) & 0.51 & 0.93 & $<0.06$ & 16.9 & 0.130 & 5.59 & 0.030 & 0.014 & 0.021 & 0.920 & $<0.005$ & 0.170 & 0.0020 & 0.0049 \\
\hline & & & & & & & & & & & & & & & \\
\hline 41 & ER-3-1 Well, NTS, Area 3, (10/96) & 0.52 & 0.99 & $<0.06$ & 17.3 & 0.160 & 10.80 & $<0.03$ & 0.043 & $<0.01$ & 0.970 & $<0.01$ & 0.180 & $<0.001$ & 0.0030 \\
\hline 42 & UE-16f Well, NTS, Area 16 & 0.045 & 0.42 & $<0.06$ & 2.1 & $\leq 0.01$ & 0.15 & $<0.01$ & $<0.001$ & $<0.007$ & 0.550 & 0.108 & 0.504 & nd & nd \\
\hline 43 & UE-1c Woll, NTS, Area 1, 411 mbsl & 0.01 & 180 & $<0.08$ & 44.3 & $<0.03$ & $<0.02$ & $<0.05$ & $<0.10$ & $<0.10$ & 0.410 & $<0.02$ & 90 & 0.0009 & 0.0041 \\
\hline 44 & UE-1c Well, NTS, Area 1, $499 \mathrm{mbsl}$ & 0.01 & 180 & $<0.06$ & 44.5 & $<0.03$ & $<0.02$ & $<0.05$ & $<0.10$ & $<0.10$ & 0.420 & $<0.02$ & 100 & $<0.0008$ & 0.0044 \\
\hline \multirow[t]{2}{*}{45} & UE-1b Woll, NTS, Area 1 & 0.01 & 180 & $<0.06$ & 37.8 & $<0.03$ & $\leq 0.02$ & $<0.05$ & $<0.10$ & $<0.10$ & 0.470 & $<0.02$ & 120 & $<0.0008$ & 0.0043 \\
\hline & & & & & & & & & & & & & & & \\
\hline 46 & UE-1a Well, NTS, Area 1 & 0.02 & 230 & $<0.06$ & 9.0 & 1 & 1.18 & $<0.05$ & $<0.10$ & $<0.10$ & 0.630 & $\leq 0.02$ & 450 & $<0.0008$ & $<0.0006$ \\
\hline 47 & ER-30-1 Well \#1, NTS, Area 30 (1/95) & 0.09 & 0.12 & $<0.06$ & 11.9 & $<0.01$ & 0.30 & $<0.01$ & 0.009 & 0.001 & 0.007 & 0.004 & $<0.002$ & $<0.0003$ & 0.0016 \\
\hline 48 & ER-30-1 Well \#2, NTS, Area 30 (2/95) & 0.083 & 0.16 & 0.06 & 14.1 & 0.020 & 0.32 & $\leq 0.01$ & 0.015 & 0.001 & 0.012 & 0.005 & $<0.002$ & 0.0003 & 0.0019 \\
\hline 49 & ER-30-1 Well \#1, NTS, Area 30 (6/96) & 0.07 & 0.11 & $<0.08$ & 12.5 & 0.013 & 0.15 & $<0.03$ & 0.009 & $<0.01$ & 0.007 & 0.018 & 0.001 & $<0.001$ & 0.0020 \\
\hline \multirow[t]{2}{*}{50} & ER-30-1 Well \#2, NTS, Area 30 (6/96) & 0.07 & 0.15 & $\leq 0.06$ & 12.7 & 0.015 & 0.16 & $<0.03$ & 0.010 & $<0.01$ & 0.009 & 0.028 & 0.001 & $<0.001$ & 0.0020 \\
\hline & & & & & & & & & & & & & & & \\
\hline 51 & U3-on \#5 Well, NTS, Area 3 & 0.06 & 0.17 & 0.04 & 16.7 & 0.040 & 1.64 & $<0.03$ & 0.043 & $<0.01$ & 0.230 & $<0.01$ & 0.160 & $<0.001$ & $<0.001$ \\
\hline 52 & UE-1q Woll, NTS, Area 1 & 0.01 & 140 & 120 & 23.8 & $<0.03$ & $<0.02$ & $<0.05$ & $<0.10$ & $<0.10$ & 0.140 & $<0.02$ & 40 & 0.0089 & 0.0023 \\
\hline 53 & U3-cn PS \#2 Well, NTS, Area 3 & 0.04 & 0.24 & 0.28 & 27.1 & 0.040 & 0.04 & $<0.03$ & 0.018 & $<0.01$ & 0.020 & $<0.01$ & $<0.01$ & 0.0020 & 0.0110 \\
\hline 54 & UE-16d Well, NTS, Area 16 & $<0.10$ & 0.22 & $<0.005$ & 13.0 & 0.003 & $<0.02$ & 0.004 & 0.002 & $<0.004$ & 0.434 & 0.002 & 0.135 & 0.0004 & 0.0016 \\
\hline \multirow[t]{2}{*}{55} & Test Well D, NTS, Area 4 & $<0.10$ & 0.28 & $<0.005$ & 21.8 & 0.023 & 1.00 & $<0.001$ & 0.014 & $<0.004$ & 0.125 & 0.008 & 0.019 & 0.0025 & 0.0007 \\
\hline & & & & & & & & & & & & & & & \\
\hline 56 & UE-17a Well, NTS, Area 17, 253 mbsl & $<0.10$ & 0.43 & $\leq 0.005$ & 5.5 & 0.063 & 0.18 & 0.002 & 0.0004 & $<0.004$ & 0.829 & 0.003 & 0.039 & $<0.0004$ & 0.0004 \\
\hline 57 & UE-17a Well, NTS, Area 17, $302 \mathrm{mbs}$ & $<0.10$ & 0.47 & $<0.005$ & 5.4 & 0.045 & 0.18 & 0.001 & 0.001 & $<0.004$ & 0.855 & 0.003 & 0.035 & $<0.0004$ & 0.0008 \\
\hline 58 & UE-17a Well, NTS, Area 17, $331 \mathrm{mbsl}$ & $<0.10$ & 0.61 & $\leq 0.005$ & 6.6 & 0.011 & $<0.02$ & $<0.001$ & 0.001 & $<0.004$ & 0.314 & 0.002 & 0.047 & 0.0004 & $<0.0004$ \\
\hline 59 & UE-18r. Well, NTS, Area 18 & 0.08 & 100 & $<0.06$ & 21.6 & $\leq 0.03$ & $<0.02$ & $<0.05$ & $<0.10$ & $<0.10$ & 0.080 & $<0.01$ & 20 & $<0.010$ & 0.0035 \\
\hline \multirow[t]{2}{*}{60} & Test Well 1, NTS, Area 17 & 0.02 & $<0.06$ & $<0.06$ & 9.1 & $<0.03$ & 0.02 & $<0.05$ & $<0.10$ & $<0.10$ & 0.010 & $<0.02$ & $<0.01$ & $<0.0008$ & $<0.0006$ \\
\hline & & & & & & & & & & & & & & & \\
\hline 61 & Water Well 8, NTS, Area 18 & $\leq 0.10$ & 0.07 & $<0.005$ & 20.8 & 0,002 & $<0.02$ & $<0.001$ & 0.003 & $<0.004$ & 0.01 & 0.001 & 0.001 & $<0.0004$ & 0.0006 \\
\hline 62 & ER-12-1 Well, NTS, Area 12 & 2.56 & 0.10 & $<0.06$ & 11.8 & 0.049 & 1.03 & 0.005 & 0.448 & 0.002 & 0.199 & 0.022 & 0.011 & 0.0002 & 0.0048 \\
\hline 63 & UE-10/ Composite, NTS, Area 8 (10/93) & 0.238 & 0.36 & $<0.06$ & 18.8 & 0.118 & 0.87 & 0.043 & 0.005 & $<0.01$ & 0.432 & 0.002 & 0.135 & 0.0022 & 0.0059 \\
\hline 64 & UE-10l Zone 1, NTS, Area 8 (3/97) & 0.33 & 0.55 & $<0.06$ & 20.0 & 0.080 & 0.88 & $<0.03$ & 0.030 & $<0.01$ & 0.470 & $<0.01$ & 0.100 & $<0.001$ & 0.0050 \\
\hline \multirow[t]{2}{*}{65} & UE-10] Zone 2, NTS, Area 8 (3/97) & 0.15 & 0.33 & $<0.06$ & 17.0 & 0.100 & 0.09 & $<0.03$ & 0.018 & $<0.01$ & 0.320 & $<0.01$ & 0.070 & $<0.001$ & 0.0030 \\
\hline & & & & & & & & & & & & & & & \\
\hline 66 & UE-10] Zone 3, NTS, Area 8 (3/97) & 0.09 & 0.25 & $\leq 0.08$ & 16.0 & 0.070 & 0.09 & $<0.03$ & 0.016 & $<0.01$ & 0.270 & $<0.01$ & 0.050 & $<0.001$ & 0.0030 \\
\hline 67 & ER-20-5 \#3, NTS, Area 20 (7/96) & 0.07 & 0.12 & 2.09 & 27.6 & 0.040 & 0.57 & $<0.03$ & 0.006 & $<0.01$ & 0.030 & $\leq 0.01$ & $\leq 0.01$ & 0.0030 & 0.0032 \\
\hline 68 & ER-20-5 \#3, NTS, Area 20 (4/97) & 0.07 & 0.11 & 3.64 & 29.0 & 0.080 & 1.24 & $<0.03$ & 0.028 & $<0.01$ & 0.020 & $<0.01$ & 0.010 & 0.0070 & 0.0040 \\
\hline 69 & ER-20-5 \#1, NTS, Area $20(6 / 96)$ & 0.092 & 0.28 & 0.20 & 28.7 & 0.004 & 0.04 & $<0.03$ & 0.007 & $<0.01$ & 0.012 & 0.020 & 0.001 & $<0.001$ & 0.0150 \\
\hline 70 & ER-20-5 \#1, NTS, Area 20 (4/97) & 0.09 & 0.27 & 3.10 & 20.0 & 0.020 & 0.39 & $<0.03$ & 0.042 & $<0.01$ & 0.020 & $<0.01$ & $<0.01$ & 0.0010 & 0.0140 \\
\hline
\end{tabular}




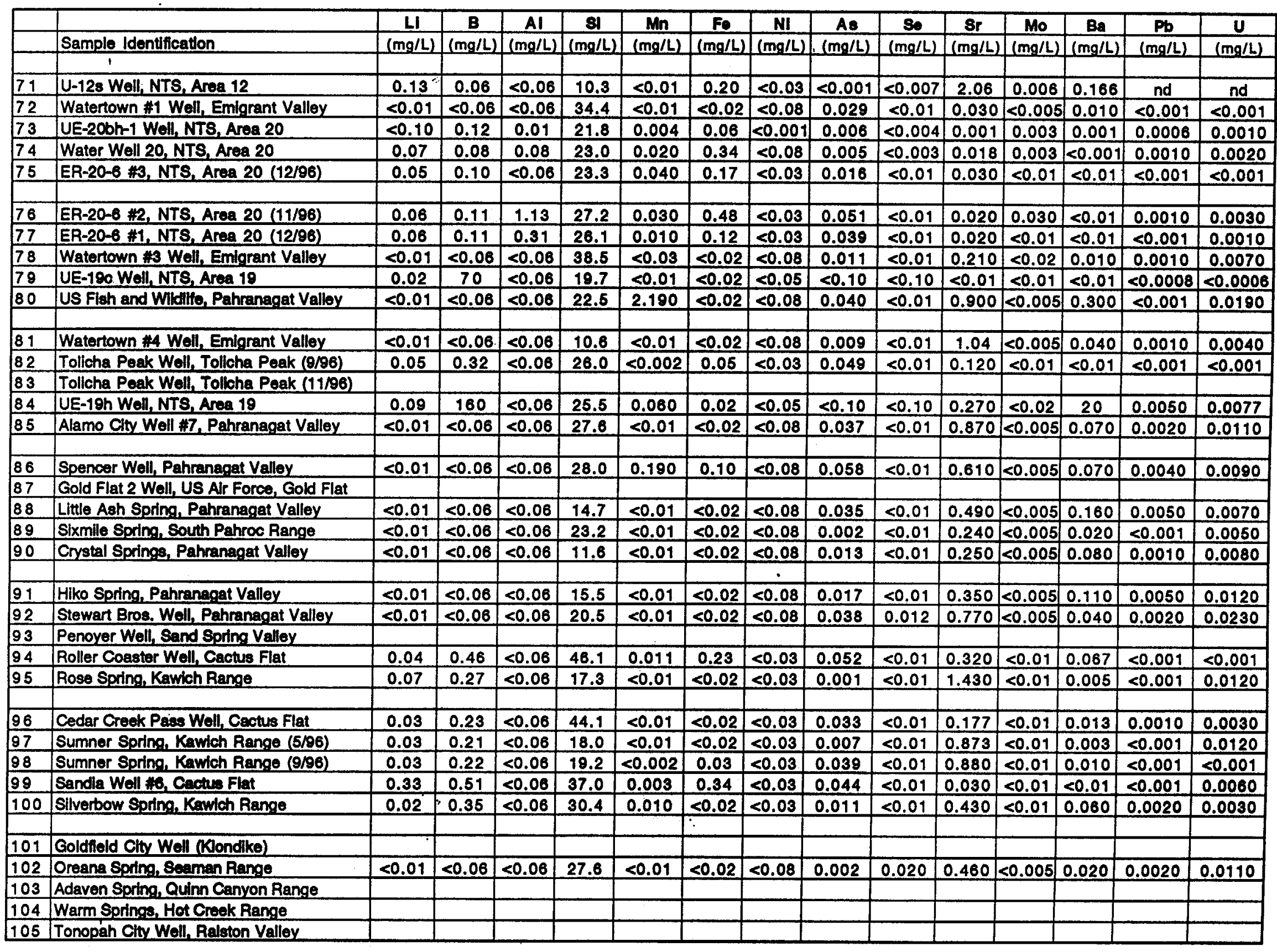


Table 3. Trace element chemistry

\begin{tabular}{|c|c|c|c|c|c|c|c|c|c|c|c|c|c|c|c|}
\hline & & 4 & B & Al & 8I & $\operatorname{Mn}$ & Fe & MI & $A B$ & 80 & $s r$ & Mo & $\mathrm{Ba}$ & $\mathbf{P b}$ & $\mathbf{U}$ \\
\hline 108 & vin Sorhoo Ranch Well Panceke Ranoo & & & & & & & & & & & & & & \\
\hline $\begin{array}{l}107 \\
10\end{array}$ & $\begin{array}{l}\text { I win sponing: Kanon Well, Panceake Range } \\
\text { Sharo Ranch Well Raltroed Valley }\end{array}$ & & & & & & & & & & & & & & \\
\hline
\end{tabular}

107 Sharp Ranch Well, Rallroed Valley 
Table 4. Isotoplc Data

\begin{tabular}{|c|c|c|c|c|c|c|c|c|c|}
\hline & Sample Identification & $8^{18} 0$ & 80 & $8^{13} \mathrm{C}$ & ${ }^{14} \mathrm{C}$ & ${ }^{14} \mathrm{C}$ apparent age & ${ }^{36} \mathrm{CV} / \mathrm{Cl}_{\text {lotal }}$ & ${ }^{87} \mathrm{Sr} /{ }^{86} \mathrm{Sr}$ & ${ }^{294} \mathrm{U} /{ }^{238} \mathrm{U}$ \\
\hline & & $\left(x_{0}\right)$ & $\left(\$_{0}\right)$ & $\left(x_{0}\right)$ & (pmc) & $\because(y r)$ & $\left(\times 10^{-13}\right)$ & & \\
\hline & & & & & & & & & \\
\hline 1 & Shoshone Spring, Shoshone CA & -12.6 & -96 & & & & & & \\
\hline 2 & Big Sping, Ash Meadows (9/95) & -13.8 & -98 & -2.6 & 3.0 & 28987 & 3.868 & 0.71709 & \\
\hline 3 & Big Spring, Ash Meadows (2/97) & -13.2 & -103 & & & & & & \\
\hline 4 & Point of Rocks Spring, Ash Moadows & -13.7 & & & & & & & \\
\hline 5 & Cold Croek Spring, Spring Mountains & -13.9 & & & & & & & \\
\hline 6. & Crystal Pool Spring, Ash Meadows (9/95) & -13.6 & -101 & -3.1 & & & 4.372 & & \\
\hline 7 & Crystal Pool Spring, Ash Meadows (2/97) & -13.7 & & & & & & & \\
\hline 8 & Well, Fumace Creek, Death Valley, CA & -13.6 & -103 & & & & & & \\
\hline 9 & Flve Springs Group, Ash Meadows & -13.8 & -102 & & & & & & \\
\hline 10 & Longstreot Spring, Ash Meadows & -13.7 & & & & & & & \\
\hline 11 & Fairbanks Spring, Ash Meadows & -13.7 & -104 & -2.4 & 2.5 & 30528 & 4.215 & 0.71272 & \\
\hline 12 & Garey Woll, Amargosa Valley & -13.2 & -100 & & & & & & \\
\hline 13 & Indlan Springs Serwer Co. Well \#1, Indlan Springs & -13.4 & & & & & & & \\
\hline 14 & USAF Well *2, Indlan Springs & -13.5 & & & & & & & \\
\hline 15 & Army Well *1, NTS, Area 22 & -13.5 & -100 & -7.1 & 5.4 & 24128 & 4.227 & 0.71196 & \\
\hline & & & & & & & & & \\
\hline$\frac{16}{17}$ & $\begin{array}{l}\text { Well, Jot. Hwy } 95 \text { and } 373 \text {, Amargosa Valley } \\
\text { J-12 Well, NTS, Area } 25\end{array}$ & -13.1 & & & & & & & \\
\hline 17 & J-12 Well, NTS, Area 25 & -13.1 & -96 & & 29.4 & 10128 & 5.030 & 0.71162 & \\
\hline 18 & Noco Woll, Amargosa Desert & -14.1 & & & & & & & \\
\hline 19 & Water Well 5c, NTS, Area 5 & -14.1 & -105 & -6.0 & 3.1 & 28637 & 6.961 & 0.71074 & \\
\hline 20 & J-13 Well, NTS, Area 25 & -13.6 & -95 & & 29.3 & 10151 & 5.023 & 0.71155 & \\
\hline & & & & & & & & & \\
\hline$\frac{21}{22}$ & UE-5n Woll, NTS, Area 5 & -13.5 & -110 & & & & 422.5 & 0.70931 & \\
\hline 22 & UE-50 Well, NTS, Area 5 & -13.8 & -105 & -7.5 & 6.7 & 22345 & 6.127 & 0.70975 & \\
\hline 23 & PW-1 Well, NTS, Aree 5 & -13.8 & -107 & -7.9 & 16.7 & 14790 & 8.415 & 0.71017 & \\
\hline 24 & PW-2 Well, NTS, Area 5 & -13.8 & -106 & -8.1 & 33.8 & 8979 & 5.274 & 0.71116 & \\
\hline 25 & PW-3 Well, NTS, Area 5 & -13.5 & -102 & -8.5 & 21.0 & 12897 & 6.779 & 0.71082 & \\
\hline 26 & Specle Spring, Bare Mountain & -12.8 & -96 & & & & & & \\
\hline 27 & Well, Boatty 12S/47E-7dbd & -14.2 & & & & & & & \\
\hline 28 & Water Well 4, NTS, Area 6 & -12.5 & & & 18.2 & 14068 & 6.161 & 0.71024 & \\
\hline 29 & Revert Soring, Oasis Valloy & -14.2 & -108 & -4.3 & 24.7 & 11673 & 4.939 & & \\
\hline 30 & Water Well C-1, NTS, Area 6 & -14.0 & -110 & -3.1 & 1.0 & 37825 & 1.663 & 0.71506 & \\
\hline & & & & & & & & & \\
\hline 31 & Water Well C, NTS, Area 6 & -13.8 & -106 & -4.2 & 0.6 & 42155 & 1.756 & 0.71503 & \\
\hline 32 & Balley Hot Spring, Oasis Valley & -14.6 & -107 & & 18.4 & 14012 & 4.845 & & \\
\hline 33 & Test Well B, NTS, Area B & -13.3 & -100 & -10.2 & 20.3 & 13169 & 7.998 & 0.70968 & \\
\hline 34 & ER-6-1 Woll, NTS, Area 6 & -14.0 & -110 & -0.7 & 2.1 & 32015 & 3.923 & 0.71283 & 0.000229 \\
\hline 35 & Goss Spring, Oasis Valley & -14.7 & -112 & & 20.8 & 13000 & 4.880 & 0.71050 & \\
\hline
\end{tabular}


Table 4. botopic Data

\begin{tabular}{|c|c|c|c|c|c|c|c|c|c|}
\hline & Sample Identifleation & $8^{10} 0$ & 80 & $8^{13} \mathrm{C}$ & ${ }^{14} \mathrm{C}$ & ${ }^{14} \mathrm{C}$ apparent age & ${ }^{36} \mathrm{CV} \mathrm{Cl}_{\text {lotal }}$ & ${ }^{87} \mathrm{Sr} /{ }^{96} \mathrm{Sr}$ & ${ }^{234} \mathrm{U}^{238} \mathrm{U}$ \\
\hline & & $\left(\psi_{0}\right)$ & $\left(\%_{0}\right)$ & $\left(\omega_{0}\right)$ & (pmo) & $(y r)$ & $\left(\times 10^{-13}\right)$ & & \\
\hline & & & & & & & & & \\
\hline 36 & UE-1h Woll, NTS, Area 1, $603 \mathrm{mbsl}$ & -13.7 & .105 & -12.1 & 12.2 & 17384 & 1.516 & 0.70924 & \\
\hline 37 & UE-1h Well, NTS, Area 1, $651 \mathrm{mbsl}$ & -13.8 & .104 & -10.3 & 23.9 & 11842 & 1.713 & 0.70955 & \\
\hline 38 & Coffers Ranch, Windmill, Oasis Valley & -12.8 & & & & & & & \\
\hline 39 & Unnamed Spring, Oasis Valloy & -13.4 & -104 & & & & & & \\
\hline 40 & ER-3-1 Well, NTS, Area 3, (9/95) & & & & 0.6 & 42155 & 1.295 & 0.71823 & 0.000208 \\
\hline 41 & ER-3-1 Well, NTS, Area 3, (10/96) & -14.2 & -112 & -3.2 & 0.8 & 39811 & 1.319 & 0.71825 & 0.000209 \\
\hline 42 & UE-16f Well, NTS, Area 16 & -13.5 & -104 & -11.7 & 3.4 & 27928 & 3.115 & 0.71136 & \\
\hline 43 & UE-1c Well, NTS, Area 1, $411 \mathrm{mbsl}$ & -13.8 & -104 & -4.6 & 2.6 & 30331 & 7.137 & 0.70988 & \\
\hline 44 & UE-1c Well, NTS, Area 1, $499 \mathrm{mbsl}$ & -13.8 & -104 & -5.3 & 2.6 & 30331 & 7.220 & 0.70988 & \\
\hline 45 & UE-1b Weil, NTS, Area 1 & & -105 & -4.5 & 16.0 & 15144 & 6.257 & 0.70953 & \\
\hline 46 & UE-1 a Well, NTS, Area 1 & .13 .5 & -103 & -8.6 & 60.5 & 4153 & 8.633 & 0.70959 & \\
\hline 47 & ER-30-1 Well, String 11 , NTS, Area 30 (1/95) & & & & 43.6 & 6862 & 5.55 & 0.70824 & 0.000137 \\
\hline 48 & ER-30-1 Well, String $\# 2$, NTS, Area 30 (2/95) & & & & 33.9 & 8942 & 5.38 & 0.70795 & 0.000107 \\
\hline 49 & ER-30-1 Well, String \#1, NTS, Area 30 (6/96) & & & -7.4 & 44.7 & 6660 & 5.202 & 0.70829 & 0.000135 \\
\hline 50 & ER-30-1 Well, String 2 , NTS, Area 30 (6/96) & & & & 44.5 & 6693 & 5.319 & 0.70825 & 0.000142 \\
\hline 51 & U3-en 45 Well, NTS, Area 3 & -14.1 & -106 & -6.1 & 3.2 & 28454 & $4: 079$ & 0.71321 & 0.000193 \\
\hline 52 & UE-1q Well, NTS, Area 1 & -14.0 & & -2.4 & 7.7 & 21195 & 7.9 & 0.71129 & 0.000268 \\
\hline 53 & U3-an PS :2 Well, NTS, Area 3 & -14.0 & .103 & -7.5 & 51483 & >modern & 12428 & 0.70962 & 0.000165 \\
\hline 54 & UE-16d Well, NTS, Area 16 & -12.9 & -95 & -10.1 & 8.0 & 20890 & 6.355 & 0.71011 & \\
\hline 55 & Test Well D, NTS, Area 4 & -14.3 & & -5.5 & 2.8 & 29707 & 7.236 & 0.71218 & \\
\hline 56 & UE-17a Well, NTS, Area 17, $253 \mathrm{mbsl}$ & -13.3 & -100. & -9.9 & 4.9 & 24999 & 5.850 & 0.71020 & \\
\hline 57 & UE-17a Well, NTS, Area 17, $302 \mathrm{mbsl}$ & -13.3 & -100 & -10.4 & 4.0 & 26630 & 5.659 & 0.71021 & \\
\hline 58 & UE-17a Well, NTS, Area 17, $331 \mathrm{mbsl}$ & -12.6 & & -11.4 & 0.7 & 40901 & 3.577 & 0.71011 & \\
\hline 59 & UE-18r Well, NTS, Area 18 & -14.7 & -110 & -1.4 & 8.2 & 20675 & 6.360 & 0.70909 & \\
\hline 60 & Test Well 1, NTS, Area 17 & -14.7 & & -10.2 & 30.1 & 9934 & 9.681 & 0.70893 & \\
\hline 61 & Water Well 8, NTS, Area 18 & -13.3 & & -9.5 & 24.9 & 11506 & 5.826 & 0.71027 & \\
\hline 62 & ER-12-1 Woll, NTS, Area 12 & .12 .5 & & -9.2 & 10.7 & 18483 & 50.6 & 0.71175 & 0.000275 \\
\hline 63 & UE-10 W Well, Composite, NTS, Area 8 (10/93) & -13.4 & & -0.1 & 3.3 & 28300 & 2.76 & 0.71451 & 0.000274 \\
\hline 64 & UE-10i Well, Zone 1, NTS, Area 8 (3/97) & .13 .6 & & -4.6 & 7.5 & 21446 & & 0.71464 & 0.000277 \\
\hline 65 & UE-10] Well, Zone 2, NTS, Area 8 (3/97) & -13.2 & & -6.4 & 11.4 & 17937 & & 0.71352 & 0.000273 \\
\hline 66 & UE-10i Well, Zone 3, NTS, Area 8 (3/97) & -12.8 & & -7.9 & 12.6 & 17150 & & 0.71261 & 0.000271 \\
\hline 67 & ER-20-5 $\because 3$ Well, NTS, Area $20(7 / 96)$ & -15.1 & -114 & -5.7 & 1450 & smodern & 173 & 0.70849 & 0.000157 \\
\hline 68 & ER-20-5 :3 Well, NTS, Area 20 (4/97) & -15.1 & & -5.8 & & & & & 0.000155 \\
\hline 69 & ER-20-5 11 Well, NTS, Area $20(6 / 96)$ & -14.9 & -114 & -2.3 & 28169 & smodern & 39400 & 0.71066 & 0.000188 \\
\hline 70 & ER-20-5 1 Well, NTS, Area 20 (4/97) & -15.0 & & -2.8 & & & & & 0.000166 \\
\hline
\end{tabular}


Table 4. lsotopic Data

\begin{tabular}{|c|c|c|c|c|c|c|c|c|c|}
\hline & Samplo Identification & $8^{12} 0$ & $8 D$ & $8^{13} \mathrm{C}$ & ${ }^{14} \mathrm{C}$ & ${ }^{14} \mathrm{C}$ apparent age & ${ }^{36} \mathrm{Cl} \mathrm{Cl}_{\text {total }}$ & ${ }^{87} \mathrm{Sr} / 86 \mathrm{Sr}$ & ${ }^{234} U /^{238} U$ \\
\hline & & $(8 \%)$ & $(\infty 6)$ & $(800)$ & (pmc) & $(y r)$ & $\left(\times 10^{-13}\right)$ & & \\
\hline & & & & & & & & & \\
\hline 71 & U-128 Well, NTS, Area 12 & -12.6 & -94 & & 100 & modern & 3.346 & 0.70555 & \\
\hline 72 & Watertown \#1 Well, Emigrant Valley & -14.6 & .109 & -5.3 & 12.1 & 17459 & 6.529 & 0.71566 & \\
\hline 73 & UE-20bh-1 Well, NTS, Area 20 & -14.7 & -109 & -9.2 & 21.0 & 12921 & 6.450 & & \\
\hline 74 & Water Well 20, NTS, Area 20 & & & & 9.1 & 19814 & 5.67 & 0.71119 & 0.000259 \\
\hline 75 & ER-20-6 \#3 Well, NTS, Area 20 (12/96) & .15 .0 & -109 & -7.2 & 16.3 & 14991 & 0.1519 & 0.70974 & 0.000257 \\
\hline 716 & ER-20-6 \#2 Well, NTS, Area 20 (11/96) & -15.0 & -110 & -7.3 & 1068.5 & & 82.26 & & 0.000138 \\
\hline 77 & ER-20-8 \#1 Well, NTS, Area 20 (12/96) & -15.0 & -115 & -6.7 & 344.2 & >modern & 15.55 & 0.71016 & 0.000221 \\
\hline 78 & Watertown $\# 3$ Well, Emigrant Valley & -13.7 & -105 & -6.0 & 27.0 & 10827 & 5.821 & 0.71551 & \\
\hline 79 & UE-190 Well, NTS, Area 19 & -15.0 & & -5.3 & 8.1 & 20797 & 6.257 & 0.70943 & \\
\hline \multirow[t]{2}{*}{80} & US Flah and Wildlito Well, Pahranagat Valley & -10.2 & -87 & -9.1 & 97.0 & 256 & 7.474 & & \\
\hline & & & & & & & & & \\
\hline 81 & Watertown \#4 Well, Emigrant Valley & -14.3 & -108 & -2.3 & 1.0 & 38152 & 3.622 & 0.72571 & \\
\hline 82 & Tollcha Peak Well, Tollcha Peak $(9 / 96)$ & -13.4 & -105 & & & & & & \\
\hline 83 & Tollcha Peak Well, Tolicha Peak (11/96) & -13.6 & -105 & & & & & & \\
\hline 84 & UE-19h Well, NTS, Area 19 & -14.8 & & -3.3 & 9.4 & 19546 & 4.786 & 0.71104 & \\
\hline \multirow[t]{2}{*}{85} & Alamo Ctiy Well \#7, Pahranagat Valley & -13.5 & -101 & -10.8 & 80.4 & 1800 & 7.414 & & \\
\hline & & & & & & & & & \\
\hline 88 & Little Ash Spring, Pahranagat Valley & $\frac{-12.8}{-14.2}$ & $\frac{-98}{-107}$ & -4.6 & & 22933 & & & \\
\hline 89 & Sixmlle Spring, South Pahroc Range & -13.1 & -93 & -11.9 & 8.6 & 1001 & $\frac{6.514}{7.157}$ & 0.71415 & \\
\hline \multirow[t]{2}{*}{90} & Gystal Springs, Pahranagat Valley & -14.4 & -107 & -5.7 & & & 6.425 & 0.71087 & \\
\hline & & & & & & & & & \\
\hline 91 & Hiko Spring, Pahranagat Valley & -14.5 & -108 & -5.2 & 6.0 & 23313 & 6.080 & 0.71108 & \\
\hline 92 & Stewart Brothers Well, Pahranagat Valloy & .14 .4 & -107 & -5.2 & & & 7.033 & & \\
\hline 93 & Penoyer Well, Sand Spring Valley & -15.1 & -118 & -8.9 & & & & & \\
\hline 94 & Roller Coaster Well, Cactus Flat & -16.0 & -129 & -6.6 & 13.2 & 16746 & & & \\
\hline 95 & Rose Soding, Kawich Range & -12.8 & -104 & -8.7 & 66.7 & 3353 & & & \\
\hline 96 & Codar Croek Pass Well, Cactus Flat & -14.1 & -111 & -10.1 & 21.9 & 12551 & & & \\
\hline 97 & Sumner Spring, Kawich Range (5/96) & -12.5 & -103 & -8.6 & 79.1 & 1940 & & & \\
\hline 98 & Sumner Eoring, Kawich Range (9/96) & -13.3 & -107 & & & & & & \\
\hline 99 & Sendla Woll \#6, Cactus Flat & -15.4 & -122 & -6.7 & & & & & \\
\hline 100 & Silverbow Spring, Kawich Range & -13.1 & -108 & -13.7 & 110.9 & $>$ modern & & & \\
\hline 101 & Goldifeld City Well (Klondike) & -14.1 & & -10.7 & & & & & \\
\hline 102 & Oreana Sprlng, Seaman Range & -10.2 & -85 & -8.9 & 96.7 & 281 & 35.19 & & \\
\hline 103 & Adaven Spring, Quinn Canyon Range & -14.1 & & -10.0 & & & & & \\
\hline 104 & Warm Springs, Hot Creek Range & -14.4 & -109 & -2.8 & & & & & \\
\hline 105 & Tonopah City Well, Ralston Valloy & -14.6 & & -13.6 & & & & & \\
\hline
\end{tabular}


Tablo 4. leotopic Data

\begin{tabular}{|c|c|c|c|c|c|c|c|c|c|}
\hline & Samplo Identfication & $8^{120} 0$ & 8D & $8^{13} \mathrm{C}$ & ${ }^{14} \mathrm{C}$ & ${ }^{14} \mathrm{C}$ apparent age & ${ }^{36} \mathrm{Cl} / \mathrm{Cl}_{\text {total }}$ & ${ }^{87} \mathrm{~S} t /{ }^{10} \mathrm{Sr}$ & ${ }^{22} U /^{252} U$ \\
\hline$i$ & & $\left(x_{0}\right)$ & $\left(\infty_{0}\right)$ & $\left(x_{0}\right)$ & (pmo) & (yr) & $\left(x 10^{-13}\right)$ & & \\
\hline & & & & & & & & & \\
\hline 106 & Twin Sorthgs Ranch Well, Pancake Range & -14.7 & & -2.8 & & & & & \\
\hline 107 & Sharp Ranch Well (Nyala), Rallroad Valley & -14.4 & -110 & -7.1 & & & & & \\
\hline
\end{tabular}


Table 4. Isotoplc Data

\begin{tabular}{|c|c|c|c|c|c|c|c|}
\hline & Sample Identification & ${ }^{4} \mathrm{He}_{\mathrm{e}}$ & ${ }^{3} \mathrm{He} /{ }^{4} \mathrm{He}$ & ${ }^{20} \mathrm{Ne}$ & ${ }^{40} \mathrm{Ar}$ & ${ }^{24} \mathrm{Kr}$ & ${ }^{132} \times \theta$ \\
\hline & & (atoms/ml) & & (atoms/ml) & (atoms $/ \mathrm{ml}$ ) & (atoms $/ \mathrm{ml}$ ) & (atoms $/ \mathrm{ml})$ \\
\hline & & & & & & & \\
\hline 1 & Shoshone Spring, Shoshone CA & & & & & & \\
\hline 2 & Bi- Spring, Ash Meadows (9/95) & $4.40 E+13$ & & $4.07 E+12$ & $6.50 \mathrm{E}+15$ & $8.24 E+11$ & $5.16 \mathrm{E}+10$ \\
\hline 3 & Blg Spring, Ash Meadows (2/97) & & & & & & \\
\hline 4 & Point of Rocks Spring, Ash Meadows & & & & & & \\
\hline 5 & Cold Creok Spring, Spring Mountains & & & & & & \\
\hline 6 & Crystal Pool Spring, Ash Meadows (9/95) & $5.91 E+12$ & & $4.08 E+12$ & $6.69 E+15$ & $8.10 E+11$ & $5.34 E+10$ \\
\hline 7 & Crystal Pool Spring, Ash Meadows (2/97) & & & & & & \\
\hline 8 & Well, Fumace Croek, Death Valley, CA & & & & & & \\
\hline 8 & Five Springs Group, Ash Meadows & & & & & & \\
\hline 10 & Longastreet Spring, Ash Meadows & & & & & & \\
\hline 11 & Falibanks Spring, Ash Meadows & $5.07 E+12$ & & $4.27 E+12$ & $6.73 E+15$ & $8.13 E+11$ & $5.47 E+10$ \\
\hline 12 & Garoy Well, Amaroosa Valley & & & & & & \\
\hline 13 & Indian Springs Sewer Co. Well \#1, Indian Springs & & & & & & \\
\hline 14 & USAF Well *2, Indlian Springs & & & & & & \\
\hline 15 & Army Woll $\# 1$, NTS, Aroa 22 & $9.91 E+12$ & & $4.30 E+12$ & $7.36 E+15$ & $9.08 E+11$ & $6.30 E+10$ \\
\hline 16 & Well, Jet. Hwy 95 and 373 , Amargosa Valley & & & & & & \\
\hline 17 & J-12 Well, NTS, Area 25 & $1.98 E+12$ & & $4.06 E+12$ & $6.90 \mathrm{E}+15$ & $8.57 E+11$ & $5.72 \mathrm{E}+10$ \\
\hline 18 & Neco Well, Amargosa Desert & & & & & & \\
\hline 19 & Water Well 5c, NTS, Area 5 & $8.69 E+12$ & & $4.54 \mathrm{E}+12$ & $8.42 E+15$ & $1.12 E+12$ & $7.33 E+10$ \\
\hline 20 & J-13 Woll, NTS, Area 25 & $4.09 E+12$ & & $4.04 E+12$ & $6.67 E+15$ & $8.24 \mathrm{E}+11$ & $5.60 E+10$ \\
\hline & & & & & & & \\
\hline 21 & UE-6n Well, NTS, Area 5 & & & & & & \\
\hline 22 & UE- 5 c Well, NTS, Area 5 & $2.25 E+13$ & & $4.26 E+12$ & $7.74 E+15$ & $1.00 E+12$ & $6.51 E+10$ \\
\hline 23 & PW-1 Well, NTS, Area 5 & & & & & & \\
\hline 24 & PW-2 Well, NTS, Area 5 & & & & & & \\
\hline 25 & PW-3 Well, NTS, Area 5 & & & & & & \\
\hline & & & & & & & \\
\hline 26 & Specle Spring, Bare Mountain & & & & & & \\
\hline 27 & Well, Beatty 12S/47E-7dbd & & & & & & \\
\hline 28 & Water Well 4, NTS, Area 6 & $3.10 E+12$ & & $4.11 E+12$ & $7.15 E+15$ & $9.38 E+11$ & $5.93 E+10$ \\
\hline 29 & Revert Spring, Oasis Valley & & & & & & \\
\hline 30 & Water Well C-1, NTS, Area 6 & $7.81 E+12$ & & $3.29 E+12$ & $4.89 E+15$ & $5.90 E+11$ & $4.12 E+10$ \\
\hline 31 & Water Well C, NTS, Area 6 & $7.99 E+12$ & & $3.33 E+12$ & $5.01 E+15$ & $5.92 E+11$ & $4.15 E+10$ \\
\hline$\frac{31}{32}$ & Balley Hot Spring, Oasis Valley & $2.73 E+13$ & & $3.48 E+12$ & $6.02 E+15$ & $7.62 E+11$ & $5.10 E+10$ \\
\hline 33 & Test Well B, NTS, Area 6 & & & & & & \\
\hline 34 & ER-6-1 Well, NTS, Area 6 & $1.03 E+13$ & 8.65E-07 & $7.22 E+12$ & $9.25 E+15$ & $1.12 E+12$ & $7.20 E+10$ \\
\hline 35 & Goss Spring, Oasis Valley & $1.56 \mathrm{E}+12$ & & $3.92 E+12$ & $7.00 E+15$ & $8.72 E+11$ & $5.69 E+10$ \\
\hline
\end{tabular}


Tablo 4. lootople Data

\begin{tabular}{|c|c|c|c|c|c|c|c|}
\hline & Semple Identification & A $\mathrm{He}$ & ${ }^{3} \mathrm{He} / \mathrm{He}$ & ${ }^{20} \mathrm{Ne}$ & ${ }^{40} \mathrm{Ar}$ & ${ }^{m} \mathrm{Kr}$ & ${ }^{132} \times 0$ \\
\hline & & (atoms $/ \mathrm{ml}$ ) & & (atoms/ml) & (atoms/ml) & (atoms/ml) & (atoms $/ \mathrm{ml}$ ) \\
\hline & & & & & & & \\
\hline 36 & UE-1h Woll, NTS, Area 1, $603 \mathrm{mbel}$ & & & $i$ & & & \\
\hline 37 & UE-1h Woll, NTS, Area 1, $651 \mathrm{mbel}$ & & & $i$ & & & \\
\hline 38 & Coffere Ranch, Windmill, Oasis Valloy & & & & & & \\
\hline 39 & Unnamed Spring, Oasis Valley & & & & & & \\
\hline 40 & ER-3-1 Well, NTS, Area 3, (9/95) & $4.89 E+13$ & 1.90E-06 & $2.99 E+12$ & $4.25 E+15$ & $5.09 E+11$ & $3.71 E+10$ \\
\hline 41 & ER-3-1 Well, NTS, Area 3, (10/96) & $3.66 E+13$ & $1.90 \mathrm{E}-0 \mathrm{~B}$ & & & & \\
\hline 42 & UE-16f Well, NTS, Area 16 & & & & & & \\
\hline 43 & UE-1c Woll, NTS, Area 1, 411 mbel & & & & & & \\
\hline 44 & UE-1c Well, NTS, Area 1, $499 \mathrm{mbsl}$ & & & & & & \\
\hline 45 & UE-1b Well, NTS, Area 1 & & & & & & \\
\hline 46 & UE-1a Well, NTS, Area 1 & & & & & & \\
\hline 47 & ER-30-1 Well, String \#1, NTS, Ares 30 (1/95) & $2.33 E+12$ & 1.12E-06 & & & & \\
\hline 48 & ER-30-1 Well, String \#2, NTS, Ares 30 (2/95) & $3.24 E+12$ & 1.08E-06 & & & & \\
\hline 49 & ER-30-1 Well, String \#1, NTS, Area 30 (6/96) & $2.24 E+12$ & $9.77 \mathrm{E}-07$ & & & & \\
\hline 50 & ER-30-1 Well, String \#2, NTS, Area $30(6 / 90)$ & $2.45 E+12$ & 9.79E-07 & & & & \\
\hline 51 & U3-en *5 Well, NTS, Area 3 & $9.08 E+13$ & $<9.96 \mathrm{E}-05$ & $5.11 E+12$ & $8.42 E+15$ & $1.10 E+12$ & $7.73 \mathrm{E}+10$ \\
\hline 52 & UE-1q Well, NTS, Area 1 & $1.84 E+12$ & $8.80 \mathrm{E}-07$ & $4.40 E+12$ & $7.98 E+15$ & $1.04 E+12$ & $7.09 E+10$ \\
\hline 53 & U3-en PS \#2 Well, NTS, Area 3 & $4.16 E+12$ & $1.61 E-01$ & & & & \\
\hline 54 & UE-16d Well, NTS, Ares 16 & & & & & & \\
\hline 55 & Test Well D, NTS, Area 4 & & & & & & \\
\hline 56 & UE-17a Woll, NTS, Area 17, $253 \mathrm{mbal}$ & & & & & & \\
\hline 57 & UE-17a Well, NTS, Area 17, $302 \mathrm{mbs}$ & & & & & & \\
\hline 58 & UE-17a Well, NTS, Area 17,331 mbsl & & & & & & \\
\hline 59 & UE-18r Well, NTS, Area 18 & & & & & & \\
\hline 60 & Test Well 1, NTS, Area 17 & & & & & & \\
\hline 61 & Water Well 8, NTS, Area 18 & & & & & & \\
\hline 62 & ER-12-1 Well, NTS, Area 12 & $4.89 E+12$ & $1.51 E-06$ & $2.55 E+13$ & $2.60 \mathrm{E}+16$ & $2.56 E+12$ & $1.38 \mathrm{E}+11$ \\
\hline 63 & UE-10/ Well, Composite, NTS, Area 8 (10/93) & $1.49 E+14$ & $1.06 \mathrm{E}-08$ & $8.14 E+12$ & $1.23 E+16$ & $1.47 E+12$ & $9.18 \mathrm{E}+10$ \\
\hline 64 & UE-10] Well, Zone 1, NTS, Area 8 (3/97) & $2.78 E+14$ & $7.72 E-07$ & & & & \\
\hline 65 & UE-10] Well, Zone 2, NTS, Area 8 (3/97) & $9.59 E+13$ & 7.63E-07 & $7.99 E+12$ & $1.10 \mathrm{E}+16$ & $1.29 \mathrm{E}+12$ & $7.83 E+10$ \\
\hline 66 & UE-10) Well, Zone 3, NTS, Aroe 8 (3197) & $6.61 \mathrm{E}+13$ & 7.67E-07 & $5.48 E+12$ & $8.58 E+15$ & $1.02 E+12$ & $6.75 E+10$ \\
\hline 87 & ER-20-5 *3 Well, NTS, Area 20 (7196) & $1.02 E+12$ & $1.86 \mathrm{E}-03$ & & & & \\
\hline 68 & ER-20-5 \#3 Well, NTS, Area 20 (4/97) & & & & & & \\
\hline 6.9 & ER-20-5 \#1 Well, NTS, Area 20 (6/96) & $6.18 E+13$ & 1.30E-01 & & & & \\
\hline 70 & ER-20-5 \#1 Well, NTS, Area 20 (4/97) & & & & & & \\
\hline
\end{tabular}


Table 4. Isotopic Data

\begin{tabular}{|c|c|c|c|c|c|c|c|}
\hline & Sample Identification & ${ }^{4} \mathrm{He}$ & ${ }^{3} \mathrm{He} /{ }^{4} \mathrm{He}$ & ${ }^{20} \mathrm{Ne}$ & ${ }^{40} \mathrm{Ar}$ & ${ }^{84} \mathrm{Kr}$ & ${ }^{132} \times \mathrm{Xe}$ \\
\hline & & (atoms $/ \mathrm{ml}$ ) & & (atoms $/ \mathrm{ml}$ ) & (atoms $/ \mathrm{ml}$ ) & (atoms/ml) & (atoms/ml) \\
\hline & & & & & & & \\
\hline 71 & U-128 Well, NTS, Area 12 & $4.27 E+12$ & & & & & \\
\hline 72 & Watertown *1 Well, Emigrant Valley & $2.13 E+12$ & & $4.04 E+12$ & $7.21 E+15$ & $9.07 E+11$ & $6.22 E+10$ \\
\hline 73 & UE-20bh-1 Well, NTS, Area 20 & & & & & & \\
\hline 74 & Water Well 20, NTS, Area 20 & $4.26 \mathrm{E}+12$ & 4.74E-07 & & & & \\
\hline \multirow[t]{2}{*}{75} & ER-20-6 \#3 Well, NTS, Area 20 (12/96) & $6.99 E+12$ & $9.27 \mathrm{E}-07$ & $1.27 E+13$ & $1.40 E+16$ & $1.43 E+12$ & $8.54 E+10$ \\
\hline & & & & & & & \\
\hline 76 & ER-20-8 \#2 Well, NTS, Area 20 (11/96) & $4.91 \mathrm{E}+12$ & $<1 E-03$ & $1.10 E+13$ & $1.44 E+16$ & $1.39 E+12$ & $8.31 \mathrm{E}+10$ \\
\hline 77 & ER-20-6 \#1 Well, NTS, Area 20 (12/96) & $1.01 E+13$ & $<1 E-03$ & $4.88 E+12$ & $7.08 E+15$ & $8.71 E+11$ & $6.14 E+10$ \\
\hline 78 & Watertown \#3 Well, Emigrant Valley & $7.08 \mathrm{E}+12$ & & $4.24 E+12$ & $7.08 E+12$ & $1.03 E+12$ & $6.89 E+10$ \\
\hline 79 & UE-19c Well, NTS, Area 19 & $8.98 E+12$ & & $3.43 E+12$ & $7.56 \mathrm{E}+15$ & $1.15 \mathrm{E}+12$ & $8.28 E+10$ \\
\hline \multirow[t]{2}{*}{80} & US Flah and Wild lite Well, Pahranagat Valley & & & & & & \\
\hline & & & & & & & \\
\hline 81 & Watertown $\# 4$ Well, Emigrant Valley & $1.32 E+14$ & & $3.96 E+12$ & $7.96 \mathrm{E}+15$ & $1.07 E+12$ & $6.91 E+10$ \\
\hline 82 & Tollcha Peak Well, Tollcha Peak (9/98) & & & & & & \\
\hline 83 & Tollche Peak Well, Tolicha Poak (11/96) & & & & & & \\
\hline 84 & UE-19h Well, NTS, Area 19 & & & & & & \\
\hline \multirow{2}{*}{85} & Alamo City Well \#7, Pahranagat Valley & $1.42 E+12$ & & $4.68 E+12$ & $8.60 E+15$ & $1.07 E+12$ & $7.17 E+10$ \\
\hline & & & & & & & \\
\hline 86 & Spencor Well, Pahranagat Valley & $1.66 E+12$ & & $5.46 E+12$ & $9.17 E+15$ & $1.18 E+12$ & $7.27 E+10$ \\
\hline 87 & Gold Flat 2 Well, US AIr Force, Gold Flat & & & & & & \\
\hline 88 & Little Ash Spring, Pahranagat Valley & $6.58 \mathrm{E}+12$ & & $3.58 E+12$ & $6.42 E+15$ & $8.35 \mathrm{E}+11$ & $5.93 E+10$ \\
\hline 89 & Stxmlle Spring, South Pahroc Range & & & & & & \\
\hline \multirow[t]{2}{*}{90} & Crystal Springs, Pahranagat Valley & $1.04 E+13$ & & $8.29 E+12$ & $9.71 E+15$ & $1.12 E+12$ & $7.62 \mathrm{E}+10$ \\
\hline & & & & & & & \\
\hline 91 & Hilko Spring, Pahranagat Valley & $7.33 E+12$ & & $4.47 E+12$ & $7.47 E+15$ & $1.18 E+12$ & $6.99 E+10$ \\
\hline 92 & Stewart Brothers Well, Pahranagat Valley & & & & & & \\
\hline 93 & Penoyer Well, Sand Spring Valley & & & & & & \\
\hline 94 & Roller Coaster Well, Cactus Flat & & & & & & \\
\hline \multirow[t]{2}{*}{95} & Rose Spring, Kawleh Range & & & & & & \\
\hline & & & & & & & \\
\hline 96 & Codar Croek Pass Well, Cactus Flat & & & & & & \\
\hline 97 & Sumner Spring, Kawich Range (5/96) & & & & & & \\
\hline 98 & Sumner Spring, Kawich Range (9/96) & & & & & & \\
\hline 99 & Sandla Well \#6, Cactus Flat & & & & & & \\
\hline 100 & Silverbow Spring, Kawich Range & & & & & & \\
\hline & & & & & & & \\
\hline 101 & Goldilield Clity Well (Klondike) & & & & & & \\
\hline 102 & Oreana Soring, Seaman Range & & & & & & \\
\hline 103 & Adaven Spring, Quinn Canyon Range & & & & & & \\
\hline 104 & Warm Spings, Hot Creek Range & & & & & & \\
\hline$\longdiv { 1 0 5 }$ & Tonooah Clty Well, Raiston Valley & & & & & & \\
\hline
\end{tabular}

105 Tonopah Clty Well, Ralston Valley 
Table 4. ketopic Data

\begin{tabular}{|c|c|c|c|c|c|c|c|}
\hline & Sample Identfication & ${ }^{4} \mathrm{He}$ & ${ }^{3} \mathrm{He} / \mathrm{He}$ & ${ }^{20} \mathrm{Ne}$ & ${ }^{40} \mathrm{Ar}$ & ${ }^{\mu} \mathrm{Kr}$ & ${ }^{132} \times 0$ \\
\hline & & (atoms/mi) & & (atoms/mi) & (atoms/ml) & (atoms/m) & (atoms/m) \\
\hline & & & & & & & \\
\hline 108 & Twhn Sprhos Ranch Well, Pancake Range & & & & & & \\
\hline 107 & Sharp Rench Well (Nyala), Railroad Valley & & & & & & \\
\hline
\end{tabular}

107 Sham Ranch Well (Nyala), Railroad Valley 
Table 5. Summary of leotople Data compllod by GooTrans, Inc.

\begin{tabular}{|c|c|c|c|c|c|c|c|c|c|}
\hline Sample Name & Location & Latitude & Longitude & Date & $8^{10} 0$ & 80 & $8^{13} \mathrm{C}$ & ${ }^{14} \mathrm{C}$ & ${ }^{14} \mathrm{C}$ app. age \\
\hline & & & & $i$ & $\left(x_{0}\right)$ & $\left(\$_{0}\right)$ & $\left(\infty_{0}\right)$ & (pmc) & (years) \\
\hline Jean Prison Well, Ivanpah Valley & sec.10, T25S, R59E & $35^{\circ} 47^{\prime} 18^{n}$ & $115^{\circ} 20^{\prime} 43^{\prime \prime}$ & $21-$ Jun-85 & -12.1 & -95 & -7.6 & 2.4 & 30830 \\
\hline Castillo Well, Goodsprings & Sec.26, T24S, R58E & $35^{\circ} 50^{\prime} 02^{\prime \prime}$ & $115^{\circ} 26^{\prime} 09^{n}$ & 21-Jun-85 & -12.8 & -94 & -9.3 & 39.4 & 7700 \\
\hline Bird Spring, Bind Spring Range & Sec.04, T24S, R59E & $35^{\circ} 53^{\prime} 20^{\prime \prime}$ & $115^{\circ} 22^{\prime} 12^{\prime \prime}$ & 23-Jun-85 & -11.7 & -88 & -7.8 & 67.5 & 3250 \\
\hline Wilson Tank, Bird Spring Range & sec.24, T23S, R58E & $35^{\circ} 56^{\prime} 02^{\prime \prime}$ & $115^{\circ} 25^{\prime} 28^{\prime \prime}$ & $22-J u n-85$ & -10.9 & -87 & -9.4 & 80.4 & 4170 \\
\hline Sky Haibor Alrport Well, Henderson & sec.03, T23S, R61E & $35^{\circ} 58^{\prime} 23^{\prime \prime}$ & $115^{\circ} 08^{\prime} 48^{\prime \prime}$ & 28-Feb-86 & -13.1 & -95 & -6.8 & 3.0 & 28990 \\
\hline Shoshone Spring, nr. Shoshone, CA & sec.30, T22S, RTE & $35^{\circ} 58^{\prime} 48^{\prime \prime}$ & $118^{\circ} 16^{\prime} 23^{\prime \prime}$ & 25-Apr-82 & -12.7 & -95 & -6.7 & 15.2 & 15570 \\
\hline Tenaya Well, Arden & SOC.27, T22S, RBOE & $36^{\circ} 00^{\prime} 42^{\prime \prime}$ & $115^{\circ} 15^{\prime} 05^{\prime \prime}$ & 10-May-83 & -12.3 & -92 & -7.4 & 99.9 & modem \\
\hline Hidden Hills Ranoh Well & 800.24, T22S, R54E & $36^{\circ} 00^{\prime} 51^{\prime \prime}$ & $115^{\circ} 51^{\prime} 51^{\prime \prime}$ & $30-J u l-87$ & -13.7 & -98 & -8.2 & 50.5 & 5650 \\
\hline Mountain Springs Fire Station & 80c.20, T22S, R58E & $36^{\circ} 01^{\prime} 08^{\prime \prime}$ & $116^{\circ} 30^{\prime} 24^{\prime \prime}$ & $30-J u l-87$ & -12.5 & -90 & -9.8 & 32.7 & 8240 \\
\hline Mountain Spring, Spring Miths. & $80 c .20, T 22 S$, R58E & $36^{\circ} 01^{\prime} 19^{\prime \prime}$ & $115^{\prime} 30^{\prime} 20^{\prime \prime}$ & 18-Jun-87 & -12.3 & -91 & & & \\
\hline Mule Spring, Spring Mths. & Boc.16, T22S, R5TE & $36^{\circ} 01^{\prime} 63^{n}$ & $115^{\circ} 34^{\prime} 48^{n}$ & 17-Jun-87 & -12.7 & -90 & & & \\
\hline Showboat Country Club well $\$ 2$ & $800.08, T 223$, R62E & $36^{\circ} 02^{\prime} 51^{\prime \prime}$ & $115^{\circ} 04^{\prime} 48^{\prime \prime}$ & $27-F o b-8 B$ & -13.3 & -97 & -7.8 & 8.0 & 20880 \\
\hline Bootleg Spring, Spring Mths. & $80 C .07, T 22 S$, R58E & $36^{\circ} 02^{\prime} 56^{\prime \prime}$ & $115^{\circ} 30^{\prime} 30^{\prime \prime}$ & 17-Jun-87 & -12.5 & -92 & & & \\
\hline Rainbow Spring, Spring Mins. & 800,07, T22S, R58E & $38^{\circ} 03^{\prime} 10^{\prime \prime}$ & $115^{\circ} 30^{\prime} 32^{\prime \prime}$ & 17-Jun-87 & -12.9 & -94 & -9.6 & 73.4 & 2560 \\
\hline Genstar Gypsum Well, Blue Dlamond & $80 C .05, T 22 S, R 59 E$ & $36^{\circ} 03^{\prime} 20^{\prime \prime}$ & $115^{\circ} 23^{\prime} 34^{\prime \prime}$ & $11-\mathrm{Jul}-88$ & -12.3 & -86 & -9.7 & 59.0 & 4360 \\
\hline & & & & & & & & & \\
\hline Sandstone Spring $\# 1$, Red Rock Canyon & $80 C .03, T 22 S, R 58 E$ & $36^{\circ} 03^{\prime} 47^{\prime \prime}$ & $115^{\circ} 28^{\prime} 09^{\prime \prime}$ & 25-Jun-85 & -12.2 & -89 & $-10.6(2)$ & $49.8(2)$ & 5760 \\
\hline Lost Cabin Spring, Spring Mtns. & sec.36, T21S, R58E & $36^{\circ} 05^{\prime} 00^{\prime \prime}$ & $115^{\circ} 39^{\prime} 08^{\prime \prime}$ & 17-Jun-87 & -12.1 & -88 & & & \\
\hline Spanish Tralls Country Club well & sec.27, T21S, RBOE & $36^{\circ} 055^{\prime} 56^{\prime \prime}$ & $115^{\circ} 15^{\prime} 38^{\prime \prime}$ & 13-Mar-86 & -12.2 & -89 & & & \\
\hline Stock Mills Well, SW Las Vegas & $\mathrm{SOC} .22, T 21 \mathrm{~S}, \mathrm{ABOE}$ & $36^{\circ} 06^{\prime} 03^{\prime \prime}$ & $115^{\circ} 15^{\prime} 41^{\prime \prime}$ & 13-Mar-86 & -12.4 & -86 & .7 .9 & 11.0 & 18250 \\
\hline Tropicana Country Club South well \#1 & $88 c .21$, T21S, R61E & $36^{\circ} 06^{\prime} 22^{\prime \prime}$ & $115^{\circ} 10^{\prime} 09^{\prime}$ & 16-May-86 & -13.2 & -95 & & & \\
\hline Pine Creek Soring, Red Rock Canyon & sec. 17, T21S, R58E & $36^{\circ} 07^{\circ} 09^{\prime \prime}$ & $115^{\circ} 29^{\prime} 52^{n}$ & 7-Aug-87 & -12.0 & -87 & & & \\
\hline Sands Hotel and Casino well \#3 & Sec.16, T21S, R61E & $36^{\circ} 07^{\prime} 28^{\prime \prime}$ & $115^{\circ} 10^{\prime} 09^{\prime \prime}$ & 19-May-86 & -13.5 & -98 & & & \\
\hline Sparkletts Drinking Water Co., Las Vegas & soc. 18, T21S, R61E & $36^{\circ} 07^{\prime} 41^{\prime \prime}$ & $115^{\circ} 11^{\prime} 45^{n}$ & 22-Apr-86 & -13.6 & -99 & & & \\
\hline BLM Visitors Center, Red Rock Canyon & Sec. 13, T21S, R5BE & $36^{\circ} 07^{\prime} 44^{\prime \prime}$ & $115^{\circ} 26^{\prime} 03^{\prime \prime}$ & 30-Jun-85 & -12.3 & -89 & -9.3 & 46.0 & 6420 \\
\hline Las Vegas Country Club woll & sec.10, T21S, R61E & $36^{\circ} 08^{\prime} 20^{\prime \prime}$ & $115^{\circ} 08^{\prime} 49^{\prime \prime}$ & 8-Jul-86 & -13.7 & -100 & & & \\
\hline & & & & & & -90 & & & \\
\hline $\begin{array}{l}\text { CC Spring, Spring Miths. } \\
\text { Red Spring, Red Rock Cenyon }\end{array}$ & $\frac{\text { sec. } 09, \text { T21S, R57E }}{\text { sec.08, T21S; R59E }}$ & $\frac{36^{\circ} 08^{\prime} 33^{\prime \prime}}{36^{\circ} 08^{\prime} 40^{\prime \prime}}$ & $\frac{115^{\bullet} 35^{\prime} 30^{n}}{116^{\bullet} 26^{\prime} \cdot 10^{n}}$ & $\frac{18-J u n-87}{-}$ & $\frac{-13.0}{-12.3(2)}$ & $-89(2)$ & $-10.5(2)$ & 62.4 & 3900 \\
\hline Cimmeron Well, Las Vegas & SOC, 04, T219, RBOE & $360^{\circ} 08^{\prime} 47^{\prime \prime}$ & $115^{\circ} 18^{\prime} 04^{\prime \prime}$ & 3-Apr-86 & -12.4 & -90 & & & \\
\hline Manse Spring; Pahrumo Valloy & $80 C, 03, T 21 S$, R54E & $36 \cdot 09^{\prime} 17^{\prime \prime}$ & $115^{\circ} 53^{\prime} 42^{\prime \prime}$ & $27-$ Jun-85 & -13.6 & -98 & -8.3 & 46.9 & 0260 \\
\hline LVWWD well \#1a & $800.35, T 203$, R60E & $360^{\prime} 09^{\prime} 36^{\prime \prime}$ & $115^{\circ} 13^{\prime} 30^{\prime \prime}$ & 14-May-86 & -13.2 & -94 & -8.3 & 17.0 & 14650 \\
\hline $\begin{array}{r}1 \\
\end{array}$ & & & & & & & & & \\
\hline Shetland Mutual Weter Usore Assoc. & $B O C .32, T 20 S$, RB1E & $36^{\circ} 09^{\prime} 39^{\prime \prime}$ & $115^{\circ} 10^{\prime} 33^{n}$ & 3-Mar-86 & -13.5 & -100 & & & \\
\hline Willow Spring (Red Rock), Spring Mins. & Se0.33, T20S, R58E & $36^{\circ} 09^{\prime} 41^{\prime \prime}$ & $115^{\circ} 29^{\prime} 51^{\prime \prime}$ & 26-Jun-85 & -12.3 & -91 & & & \\
\hline Coal Spring, Spring Mtns. & $800.31, T 20 S$, R57E & $36^{\circ} 09^{\prime} 44^{\prime \prime}$ & $115^{\circ} 37^{\prime} 12^{n}$ & 18-Jun-87 & -12.9 & -91 & & & \\
\hline LVVWD well \#112 & sec.31, T20S, R61E & $36^{\circ} 09^{\prime} 53^{\prime \prime}$ & $115^{\circ} 11^{\prime} 22^{n}$ & 13-Jun-86 & -13.4 & -97 & -7.3 & 14.0 & 18250 \\
\hline Klup Soring, Soring Mtns. & $800.31, \mathrm{~T} 20 \mathrm{~S}, \mathrm{~A} 56 \mathrm{E}$ & $36^{\circ} 09^{\prime} 56^{\prime \prime}$ & $116^{\circ} 43^{\prime} 28^{n}$ & $=$ & $-12.9(2)$ & $-93(2)$ & & & \\
\hline
\end{tabular}


Table 5. Summary of lsotople Data complled by GooTrans, Inc.

\begin{tabular}{|c|c|c|c|c|c|c|c|c|c|}
\hline Sample Name & Location & Latitude & Longltude & Date & $8^{180}$ & 80 & $8^{13} \mathrm{C}$ & ${ }^{14} \mathrm{C}$ & ${ }^{14} \mathrm{C}$ app. age \\
\hline & & & & $\therefore$ & $\left(\infty_{0}\right)$ & $(\$ \infty)$ & $\left(\phi_{0}\right)$ & (pma) & (yoars) \\
\hline Bootlegger Spr. (Gave Spr), Spring Mtns. & sec.33, T20S, R57E & $360^{\circ} 09^{\prime} 59^{\prime \prime}$ & $115^{\circ} 36^{\prime} 48^{n}$ & - & $-12.8(3)$ & $-93(3)$ & & & \\
\hline Unton Pacilic Rallroad well *3 & 800.33, T20S, R61E & $36^{\circ} 10^{\prime} 05^{\prime \prime}$ & $116^{\circ} 09^{\prime} 13^{\prime \prime}$ & $9-A p r-B B$ & -13.8 & -100 & & & \\
\hline LWWD well $\$ 7 \mathrm{a}$ & soc.31, T20S, R60E & $36^{\circ} 10^{\prime} 08^{\prime \prime}$ & $116^{\circ} 17^{\prime} 61^{\prime \prime}$ & 17-Jun-86 & -13.2 & -98 & & & \\
\hline Sky Mt. Resort, Spring Mtno. & Sec.34, T20S, R57E & $38^{\circ} 10^{\prime} 13^{\prime \prime}$ & $115^{\circ} 34^{\prime} 44^{n}$ & 28-Jun-85 & -13.3 & -96.5 & -10.1 & 84.1 & 1430 \\
\hline LVVWD well \#14 & Sec.31, T20S, R61E & $38^{\circ} 10^{\prime} 18^{\prime \prime}$ & $115^{\circ} 11^{\prime} 22^{n}$ & 6-May-86 & -13.2 & -97 & & & \\
\hline White Rock Spring, Spring Mtns. & $80 c .33$, T20S, R58E & $36^{\circ} 10^{\prime} 27^{\prime \prime}$ & $115^{\circ} 28^{\prime} 43^{\prime \prime}$ & - & $-12.6(3)$ & $-91(3)$ & -10.4 & 44.8 & 6640 \\
\hline LVVWD well \#34 & 800.29, T20S, R61E & $36^{\circ} 10^{\prime} 31^{\prime \prime}$ & $115^{\circ} 11^{\prime} 06^{\prime \prime}$ & $15-$ May-86 & -13.6 & -99 & & & \\
\hline LVVWD well \#15a & sec.30, T20S, R61E & $36^{\circ} 10^{\prime} 31^{\prime \prime}$ & $115^{\circ} 11^{\prime} 23^{\prime \prime}$ & 14-May-86 & -13.8 & -97 & & & \\
\hline LVVWD well \#16 & Sec. 30, T20S, R61E & $36^{\circ} 10^{\prime} 31^{\prime \prime}$ & $115^{\circ} 11^{\prime} 39^{\prime \prime}$ & 4-Jun-86 & -13.6 & -97 & & & \\
\hline LVVWD well \#17 & $800.30, T 20 S$, R61E & $366^{\circ} 10^{\prime} 31^{\prime \prime}$ & $115^{\circ} 11^{\prime} 39^{\prime \prime}$ & $4-\sqrt{u}$ un-86 & -13.5 & -97 & & & \\
\hline La Madre Spring, Spring Mtns. & sec.29, T20S, A58E & $36^{\circ} 111^{\prime} 01^{\prime \prime}$ & $115^{\circ} 30^{\prime} 20^{n}$ & 5-Aug-87 & -13.1 & .93 & & & \\
\hline LVVWD well \#18a. & sec.27, T20S, R60E & $36^{\circ} 11^{\prime} 12^{\prime \prime}$ & $115^{\circ} 14^{\prime} 38^{n}$ & 6-Jun-86 & -13.4 & -94 & & & \\
\hline LVVWD well \#45 & sec.19, T20S, R61E & $36^{\circ} 11^{\prime} 50^{n}$ & $116^{\circ} 12^{\prime} 13^{\prime \prime}$ & 13-May-86 & -14.0 & .101 & & & \\
\hline LVWWD woll \#22a & $800.21, T 20 S$, R60E & $36 \cdot 12$ '05" & $115^{\circ} 15^{\prime} 43^{n}$ & $4-\sqrt{u n}-86$ & -14.0 & -100 & & & \\
\hline NLVWD Robingon Well & Sec.18, T20S, R61E & $36^{\circ} 12^{\prime} 17^{\prime \prime}$ & $115^{\circ} 11^{\prime} 41^{\prime \prime}$ & $3-\sqrt{u n}-86$ & -14.1 & -100 & & & \\
\hline Pahrump Spring Well, Pahrump & Bec.14, T20S, R53E & $38^{\circ} 12^{\prime} 27^{\prime \prime}$ & $115^{\circ} 59^{\prime} 01^{\prime \prime}$ & 27-Jun-85 & -13.6 & -97 & -7.6 & 26.0 & 11135 \\
\hline Nells AFB \#13, NE Les Vegas & Sec.16, T20S, R62E & $36^{\circ} 12^{\prime} 44^{\prime \prime}$ & $115^{\circ} 03^{\prime} 00^{\prime \prime}$ & 3-Apr-BB & -13.8 & -98 & & & \\
\hline NLYWD West Cheyenne Well & Boc.18, T20S, R61E & $36^{\circ} 12^{\prime} 56^{\prime \prime}$ & $115^{\circ} 11^{\prime} 42^{\prime \prime}$ & 12-Jun-86 & -13.8 & -100 & & & \\
\hline Trout Spring, Spring Mins. & 8ec.10, T20S, R56E & $36^{\circ} 13^{\prime} 22^{\prime \prime}$ & $115^{\circ} 40^{\prime} 59^{\prime \prime}$ & - & $-13.6(22)$ & $-98(20)$ & & & \\
\hline Hartzskd Woll, Las Vegas & $800.04, T 20 S$, R61E & $36^{\circ} 14^{\prime} 03^{n}$ & $115^{\circ} 09^{\prime} 16^{n}$ & 4-Mar-86 & -12.4 & -90 & & & \\
\hline NLVWD Desert Alre Woll & 800.06, T208, R61E & $36^{\circ} 14^{\prime} 16^{\prime \prime}$ & $115^{\circ} 12^{\prime} 18^{\prime \prime}$ & 6-Mar-86 & -14.0 & .102 & & 19.0 & 13730 \\
\hline Lake Mead Base Woll *3, Las Vegas & 800.01, T20S, R62E & $36^{\circ} 14^{\prime} 21^{\prime \prime}$ & $116^{\circ} 00^{\prime} 16^{\prime \prime}$ & $29-$ Sep-86 & -13.8 & -102 & -5.3 & 5.6 & 23830 \\
\hline Echo Spring, Spring Mins. & $80 c .01$, T20S, R56E & $36^{\circ} 14^{\prime} 21^{\prime \prime}$ & $115^{\circ} 38^{\prime} 21^{\prime \prime}$ & 16-Apr-80 & -12.8 & -95 & & & \\
\hline Harris Spring, Spring Mins. & sec.01, T20S, R57E & $36^{\circ} 14^{\prime} 27^{\prime \prime}$ & $115^{\circ} 32^{\prime} 35^{\prime \prime}$ & $15-\mathrm{Jul}-87$ & -13.7 & -97 & & & \\
\hline Craig Ranch Country Club 2, Las Vegas & sec.03, T20S, R61E & $36^{\circ} 14^{\prime} 29^{\prime \prime}$ & $115^{\circ} 09^{\prime} 00^{\prime \prime}$ & 4-Mar-86 & -14.5 & -108 & & 3.0 & 28990 \\
\hline Rainbow Spring \#2, Spring Mtrs. & sec.06, T20S, R57E & $36^{\circ} 14^{\prime} 36^{\prime \prime}$ & $116^{\circ} 37^{\prime} 55^{\prime \prime}$ & 19-Aug-82 & -12.4 & -96 & -9.8 & 91.7 & 716 \\
\hline Peak Spring Canyon Crook, Spring Mins. & 806.05, T20S, R56E & $36^{\circ} 14^{\prime} 40^{\prime \prime}$ & $115^{\circ} 43^{\prime} 09^{n}$ & - & $-13.3(6)$ & $-95(6)$ & & & \\
\hline Nellis AFB \#4,;NE Las Vogas & Bec.36, T19S, R62E & $36^{\circ} 14^{\prime} 56^{\prime \prime}$ & $115^{\circ} 00^{\prime} 15^{\prime \prime}$ & 3-Apr-86 & -13.2 & -95 & -6.3 & 21.0 & 12900 \\
\hline Calneva Pipe, $N$. of Huy 604 , Las Vegas & sec.33, T19S, R62E & $36 \cdot 14^{\prime} 59^{\prime \prime}$ & $115^{\circ} 02^{\prime} 39^{n}$ & - & -13.1 & -98 & & & \\
\hline Kyle Spring, Spring Mtns. & sec.31, T19S, R57E & $36^{\circ} 15^{\prime} 00^{\prime \prime}$ & $115^{\circ} 37^{\prime} 44^{\prime \prime}$ & $21-0 \mathrm{ct}-88$ & -13.6 & .96 & & & \\
\hline Peak Spring, Spring Mitre. & sec.28, T19S, R56E & $36^{\circ} 16^{\prime} 40^{\prime \prime}$ & $115^{\circ} 41^{\prime} 49^{\prime \prime}$ & 23-Jun-87 & -13.1 & .94 & -9.5 & 97.7 & 192 \\
\hline Pat Well, NW Las Vegas & sec.25, T19S, R59E & $36^{\circ} 15^{\prime} 45^{\prime \prime}$ & $115^{\circ} 19^{\prime} 37^{n}$ & 31-Mar-87 & -12.9 & -92 & -8.5 & 11.2 & 18100 \\
\hline Kyle Canyon Huy Well 1, Spring Mtns. & sec.28, T19S, R57E & $36^{\circ} 15^{\prime} 54^{\prime \prime}$ & $115^{\circ} 35^{\prime} 69^{n}$ & $21-0 \mathrm{ct}-88$ & -14.0 & -97 & & 83.0 & 1540 \\
\hline Taylor's Steak House Restaurant & sec.22, T19S, R60E & $36^{\circ} 17^{\prime} 08^{\prime \prime}$ & $115^{\circ} 14^{\prime} 41^{\prime \prime}$ & 3-Nov-86 & -13.7 & -101 & -7.1 & 14.0 & 16250 \\
\hline Martin Well, NW Las Vegas Valley & Sec.22, T19S, R60E & $36^{\circ} 17^{\prime} 25^{\prime \prime}$ & $115^{\circ} 14^{\prime} 35^{\prime \prime}$ & 30 -Jun-85 & -13.4 & -100 & -6.7 & 1.8 & 32760 \\
\hline
\end{tabular}


Table 5. Summay of lsotoplo Data compiled by GeoTrans, Inc.

\begin{tabular}{|c|c|c|c|c|c|c|c|c|c|}
\hline Sample Name & Location & Latitude & Longitude & Date & $8^{10} \mathrm{O}$ & 80 & $8^{13} \mathrm{C}$ & ${ }^{14} \mathrm{C}$ & ${ }^{14} \mathrm{C}$ app. ago \\
\hline & & & & & $\left(x_{0}\right)$ & $\left(x_{0}\right)$ & $\left(x_{0}\right)$ & (pmc) & (years) \\
\hline Lee Canyon Sk Soring 1, Soring Mtns. & $80 c .15$, T19S, R56E & $36^{\circ} 17^{\prime} 32^{\prime \prime}$ & $116^{\circ} 41^{\prime} 03^{\prime \prime}$ & 6-Aug-87 & -13.6 & -96 & & & \\
\hline East Horse Spring, Spring Mtrs. & sec. 14, T19S, R54E & $36^{\circ} 17^{\prime} 60^{\prime \prime}$ & $116^{\circ} 52^{\prime} 15^{n}$ & $25-J u n-87$ & -13.6 & -96 & & & \\
\hline Grapevine Spring, E. Spring Mtns. & sec. 16, T19S, R58E & $36^{\circ} 18^{\prime} 03^{n}$ & $115^{\circ} 29^{\prime} 25^{\prime \prime}$ & - & -13.2 & $-92(2)$ & -9.1 & 46.0 & 6420 \\
\hline Deer Creek Spring \#1, Spring Mtns. & Sec. 12, T19S, R58E & $36^{\circ} 18^{\prime} 23^{\prime \prime}$ & $115^{\circ} 38^{\prime} 15^{\prime \prime}$ & - & $-13.9(7)$ & $-98(7)$ & & & \\
\hline Deer Creek Spring'\#2, Spring Mtns. & sec.07, T19S, R57E & $36^{\circ} 18^{\prime} 27^{\prime \prime}$ & $115^{\circ} 37^{\prime} 37^{\prime \prime}$ & 28-Jun-85 & -13.4 & -98 & -9.6 & $102(2)$ & modern \\
\hline Amargosa R. (well) nr. Death Valley Jet, CA & sec. 18, T25N, R6E & $38^{\circ} 18^{\prime} 33^{\prime \prime}$ & $116^{\circ} 22^{\prime} 00^{\prime \prime}$ & $21-$ Nov-83 & -12.7 & -98 & & 48.7 & 5950 \\
\hline Racel Well, NW Las Vegas Valley & Sec.09, T19S, R60E & $36^{\circ} 18^{\prime} 40^{\prime \prime}$ & $115^{\circ} 15^{\prime} 39^{\prime \prime}$ & $10-$ May-83 & -13.3 & -99 & -6.7 & 99.9 & modern \\
\hline Gillbert Well, N. of Hwy 157 & 800.07, T19S, R59E & $36^{\circ} 18^{\prime} 45^{\prime \prime}$ & $115^{\circ} 25^{\prime} 08^{\prime \prime}$ & 20-Aug-82 & -12.7 & -98 & -8.8 & 81.4 & 4030 \\
\hline Stewart Well, Spring Mitns. & $80 C .10$, T19S, R56E & $36^{\circ} 19^{\prime} 10^{\prime \prime}$ & $115^{\circ} 40^{\prime} 20^{\prime \prime}$ & 24-Jun-86 & -13.4 & -100 & -8.0 & 31.6 & 8520 \\
\hline Tule Spring Well, Floyd Lamb State Park & soc.09, T19S, R60E & $36^{\circ} 19^{\prime} 14^{\prime \prime}$ & $115^{\circ} 16^{\prime} 00^{\prime \prime}$ & $30-J$ un-85 & -13.4 & -99 & -7.0 & 13.8 & 16310 \\
\hline Clark Spring, Spring Mins. & 806.08, T198, R56E & $36^{\circ} 19^{\prime} 14^{\prime \prime}$ & $115^{\circ} 43^{\prime} 15^{\prime \prime}$ & 24-Jun-87 & -13.7 & -99 & & & \\
\hline Mulder Well, NW Las Vegas Valloy & sec. 01, T19S, R60E & $38^{\circ} 19^{\prime} 25^{n}$ & $115^{\circ} 13^{\prime} 22^{\prime \prime}$ & $25-J u n-86$ & -13.2 & -97 & -7.2 & 23.8 & 11870 \\
\hline Mifflin Woll, N. of Hime 157 & $80 C .01, T 198$, A5BE & $38^{\circ} 18^{\prime} 32^{\prime \prime}$ & $116^{\circ 25} \cdot 23^{\prime \prime}$ & - & $-13.4(2)$ & $-102(2)$ & -7.7 & 16.4 & 14950 \\
\hline Lee Canyon West Spring, Spring Mins. & Boc.02, T19S, R58E & $36^{\circ} 19^{\prime} 37^{\prime \prime}$ & $115^{\circ} 39^{\prime} 45^{\prime \prime}$ & 15-Jul-87 & -14.0 & -100 & & & \\
\hline Cortney Well, Hiwy 157, NW Las Vegas & SOC.02, T19S, R59E & $36^{\circ} 19^{\prime} 42^{\prime \prime}$ & $115^{\circ} 20^{\prime} 52^{\prime \prime}$ & 21-Oct-88 & -13.9 & -99 & -7.9 & 48.4 & 6000 \\
\hline & & & & & & & & & \\
\hline Adams Well, nr. Jet. Hwy 157 and 95 & Sec.01, T19S, R59E & $36^{\circ} 19^{\prime} 47^{\prime \prime}$ & $115^{\circ} 19^{\prime} 60^{\prime \prime}$ & $20-$ Aug-82 & -12.7 & -98 & -8.2 & 50.1 & 5710 \\
\hline Lee's Oasis Well, Jot. Huy 157 and 95 & 800.01, T19S, R59E & $36^{\circ} 19^{\prime} 48^{\prime \prime}$ & $115^{\circ} 18^{\prime} 55^{\prime \prime}$ & $24-J u n-88$ & -13.4 & -98 & -8.6 & 49.6 & 5800 \\
\hline Holland Well, NW Las Vogas Valley & SeC.05, T19S, R60E & $36^{\circ} 20^{\prime} 00^{n}$ & $115^{\circ} 17^{\prime} 00^{\prime \prime}$ & 20-Aug-82 & -12.6 & -98 & -6.2 & 11.3 & 18020 \\
\hline Summer Homes Well, Spring Mins. & Sec, $35, T 18 S, R 56 E$ & $36^{\circ} 20^{\prime} 06^{\prime \prime}$ & $115^{\circ} 39^{\prime} 18^{\prime \prime}$ & $26-$ Sep-86 & $-13.7(2)$ & $-100(2)$ & -10.8 & 91.1 & 770 \\
\hline Upper Macks Canyon Spr., Spring Mtns. & SOC.33, T18S, R56E & $36 \cdot 20^{\prime} 07^{\prime \prime}$ & $115^{\circ} 41^{\prime} 11^{\prime \prime}$ & 15-Jul-87 & -14.3 & -103 & & & \\
\hline Grassy Spring, Spring Mths. & SOC.32, T18S, R58E & $36^{\circ} 20^{\prime} 23^{\prime \prime}$ & $115^{\circ} 29^{\prime} 57^{\prime \prime}$ & 28-Jul-87 & -13.5 & -99 & & & \\
\hline Buck Spring, Spring Mins. & soc.35, T18S, R55E & $36^{\circ} 20^{\prime} 37^{\prime \prime}$ & $115^{\circ} 46^{\prime} 29^{\prime \prime}$ & 1-Jul-87 & -13.6 & -98 & & & \\
\hline Paiute Indian Resenvation Well & SOC.26, T18S, R59E & $36^{\circ} 21^{\prime} 02^{\prime \prime}$ & $115^{\circ} 20^{\prime} 33^{\prime \prime}$ & 21-Aug-82 & -14.0 & -98 & -8.2 & 57.0 & 4650 \\
\hline Wood Spring, Spring Mins. & sec.25, T18S, R55E & $36^{\circ} 21^{\prime} 09^{\prime \prime}$ & $115^{\circ} 44^{\prime} 39^{\prime \prime}$ & $3-J u l-88$ & -13.4 & -98 & & & \\
\hline Mud Springs, Spring Mins. & sec.22, T18S, R56E & $36^{\circ} 22^{\prime} 16^{\prime \prime}$ & $115^{\circ} 41^{\prime} 00^{\prime \prime}$ & 28-Jul-87 & -14.1 & -106 & & & \\
\hline Trough Spring, Sping Mins. & $80 C .23, T 18 S$, R55E & $38^{\circ} 22^{\prime} 38^{\prime \prime}$ & $11.5^{\circ} 46^{\prime} 28^{\prime \prime}$ & $1-J u l-87$ & -14.1 & -100 & & & \\
\hline MoFarland Spring, Spring Mins. & BeC.13, T18S, R55E & $36^{\circ} \cdot 22^{\prime} 54^{\prime \prime}$ & $116^{\circ} 44^{\prime} 38^{\prime \prime}$ & 13-Jul-87 & -13.7 & -99 & -10.8 & 84.6 & 1380 \\
\hline Wood Canyon Spring, Spring Mins. & $80 C, 08, T 18 S$, R54E & $38^{\circ} 23^{\prime} 58^{n}$ & $116^{\circ} 55^{\prime} 64^{\prime \prime}$ & 7-Jul-87 & -13.2 & -93 & & & \\
\hline Point of Rocks Springs, Ach Meadows & sec.07, T18S, R51E & $36^{\circ} 24^{\prime} 06^{n}$ & $116^{\circ} 16^{\prime} 23^{\prime \prime}$ & 8-Mar-81 & -13.6 & -102 & $-6.5(3)$ & $7.7(3)$ & 21220 \\
\hline Cold Creok Spring, Spring Mins. & $80 c .01, T 18 S$, R65E & $36^{\circ} 24^{\prime} 32^{\prime \prime}$ & $116^{\circ} 44^{\prime} 36^{\prime \prime}$ & - & $-13.8(20)$ & $-100(18)$ & & $73.4(3)$ & 2560 \\
\hline Willow Spring, Spring Mtres. & $800.02, T 18 S$, R55E & $36^{\circ} 25^{\prime} 00^{\prime \prime}$ & $115^{\circ} 45^{\prime} 47^{\prime \prime}$ & 5-Apr-88 & -13.8 & -98 & -9.6 & 79.2 & 1030 \\
\hline Young Well, NW Las Vegas Valley & $800.04, T 18 S$, R59E & $36 \cdot 25^{\prime} 11^{\prime \prime}$ & $115^{\circ} 22^{\prime} 58^{\prime \prime}$ & $25-$-Sep-8B & -12.8 & -93 & -6.7 & 13.7 & 16430 \\
\hline Crystal Pool, Ash Meadows & $80 C .03, T 188$, R R $50 E$ & $38^{\circ} 25^{\prime} 13^{\prime \prime}$ & $118^{\circ} 19^{\prime} 23^{\prime \prime}$ & - & $-13.7(2)$ & $-102(2)$ & -5.0 & 11.6 & 17810 \\
\hline Johnnie Gold Pan W'ell & $800.01, T 188$, R62E & $36^{\circ} 25^{\prime} 14^{\prime \prime}$ & $116^{\circ} 04^{\prime 1} 12^{\prime \prime}$ & $30-\mathrm{Jul}-87$ & -12.7 & -91 & -10.1 & 10.5 & 18630 \\
\hline Brooks Well, NW Las Vegas Valley & 800.04, T18S, R59E & $36^{\circ} 25^{\prime} 20^{\prime \prime}$ & $115^{\bullet} 22^{\prime} 40^{\prime \prime}$ & 24-Jun-86 & -12.7 & -93 & -6.9 & 35.7 & 8515 \\
\hline
\end{tabular}


Table 5. Summary of lsotoplo Data compiled by GeoTrans, Inc.

\begin{tabular}{|c|c|c|c|c|c|c|c|c|c|}
\hline Sample Name & Location & Latitude & Longltude & Date & $8^{18} 0$ & 80 & $8^{13} \mathrm{C}$ & ${ }^{14} \mathrm{C}$ & ${ }^{14} \mathrm{C}$ app. age \\
\hline & & & & $i \cdot$ & $(\%)$ & (\%) & $\left(x_{0}\right)$ & (pmc) & (verers) \\
\hline Shown Well, NW Las Veges Valley & $800.04, T 185$, R50E & $36^{\circ} 25^{\prime} 31^{\prime \prime}$ & $115^{\circ} 22^{\prime} 44^{\prime \prime}$ & $25-80 p-86$ & -13.0 & -94 & -8.8 & 20.4 & 13140 \\
\hline Crystal Springs, Spring Mtns. & 8ec.36, T17S, R53E & $36^{\circ} 25^{\prime} 39^{\prime \prime}$ & $115^{\circ} 58^{\prime} 25^{\prime \prime}$ & 7-Jul-87 & -12.6 & -91 & -4.8 & 10.5 & 18630 \\
\hline Corn Creek Spring, NW Las Vegas Valley & sec.34, T178, R59E & $36^{\circ} 28^{\prime} 20^{\prime \prime}$ & $15^{\circ} 21^{\prime} 26^{\prime \prime}$ & - & $-12.9(4)$ & $-94(4)$ & $-9.4(2)$ & 15.9 & 16230 \\
\hline Travertine Springs, Death Valley, CA & 800.26, T27N; R1E & $36^{\circ} 26^{\prime} 27^{\prime \prime}$ & $110^{\circ} 49^{\prime} 40^{\prime \prime}$ & 22-Apr-82 & -13.9 & -102 & -8.5 & 10.9 & 18300 \\
\hline Horseshutem Spring, Spring Mitns. & sec.27, T17S, R53E & $36^{\circ} 26^{\prime} 35^{\prime \prime}$ & $115^{\circ} 59^{\prime} 57^{\prime \prime}$ & 9-Jul-87 & -13.1 & -93 & & & \\
\hline . & & & & & & & & & \\
\hline Big Timber Spring, Spring Mins. & 8ec.29, T17S, R54E & $36^{\circ} 26^{\prime} 42^{\prime \prime}$ & $116^{\circ} 66^{\prime} 37^{\prime \prime}$ & 27-Jün-85 & -13.3 & -93 & -8.8 & 87.3 & 1120 \\
\hline Grapevine Springs, Spring Mtns. & sec. $21, T 17$, R53E & $36^{\circ} 27^{\prime} 25^{\prime \prime}$ & $116^{\circ} 01^{\prime} 32^{\prime \prime}$ & - & $-13.0(2)$ & $-92(2)$ & & & \\
\hline Texas Spring, Death Valloy, CA & sec.23, T27N,R1E & $38^{\circ} 27 \cdot 28^{\prime \prime}$ & $1160^{\circ} 0^{\prime} 11^{\prime \prime}$ & $22-A p r-82$ & -13.7 & -102 & & & \\
\hline Gold Spring, Spring Mins. & sec.24, T17S, R53E & $36^{\circ} 27^{\prime} 48^{\prime \prime}$ & $115^{\circ} 57^{\prime} 39^{\prime \prime}$ & 14-Jul-87 & -13.6 & -94 & & & \\
\hline Com Creek Ranch Well & sec.20, T17S, R59E & $36^{\circ} 27^{\prime} 52^{n}$ & $115^{\circ} 23^{\prime} 53^{\prime \prime}$ & 2-Dec-86 & -13.5 & -95 & -7.7 & 7.0 & 21980 \\
\hline Kwlchup Spring, Spring Mins. & Sec.17, T17S, R53E & $36^{\circ} 28^{\prime} 21^{\prime \prime}$ & $116^{\circ} 02^{\prime} 16^{\prime \prime}$ & 9-Jul-87 & -13.2 & -96 & & & \\
\hline Silver Flag $\alpha$-Well, Nellis AF Range & sec. 14, T175, R58E & $36^{\circ} 28^{\prime} 34^{\prime \prime}$ & $115^{\circ} 26^{\prime} 45^{\prime \prime}$ & 18-Dec-87 & -13.7 & -99 & & & \\
\hline Fairbanks Spring, Ash Meadows & sec.09, T17S, R50E & $36^{\circ} 29^{\prime} 26^{\prime \prime}$ & $116^{\circ} 20^{\prime} 29^{\prime \prime}$ & - & $-13.7(2)$ & $-104(2)$ & $-6.2(2)$ & $4.3(4)$ & 26050 \\
\hline Nevares Springs, Death Valley, CA & sec.36, T28N; R1E & $38^{\circ} 30^{\prime} 44^{\prime \prime}$ & $116^{\circ} 49^{\prime} 14^{\prime \prime}$ & 22-Apr-82 & -13.6 & -104 & -9.3 & 9.2 & 19720 \\
\hline Divide Well, Three Lakes Valley & sec.31, T16S, R58E & $36^{\circ} 30^{\prime} 45^{\prime \prime}$ & $115^{\circ} 28^{\prime} 45^{\prime \prime}$ & 26-Jul-87 & -13.7 & -98 & & & \\
\hline Indian Springs Prison Well \#1 & sec.33, T16S, R57E & $36 \cdot 30^{\prime} 52^{\prime \prime}$ & $115^{\circ} 33^{\prime} 15^{\prime \prime}$ & 26-Jun-85 & -13.7 & -102 & -6.6 & 4.9 & 24930 \\
\hline Mathew's Well, Amargosa Valley & 8ec.36, T16S, R49E & $36^{\circ} 31^{\prime} 32^{\prime \prime}$ & $116^{\circ} 24^{\prime} 00^{\prime \prime}$ & 11-May-81 & -13.7 & -104 & -4.4 & 10.9 & 18300 \\
\hline Old Dry Well, Three Lakes Valley & 80c.29, T16S, R58E & $36^{\circ} 31^{\prime} 35^{\prime \prime}$ & $115^{\circ} 28^{\prime} 14^{\prime \prime}$ & 19-Dec-87 & -13.2 & -95 & & & \\
\hline Polnt Bravo Well (Alpha Post) & 8ec.29, T165, R57E & $36^{\circ} 32^{\prime} 06^{n}$ & $115^{\circ} 33^{\prime} 55^{\prime \prime}$ & 23-Jun-86 & -13.2 & -98 & -7.1 & 6.1 & 23120 \\
\hline South Black Hills Woll & $800.26, T 16 S, R 58 E$ & $36^{\circ} 32^{\prime} 12^{\prime \prime}$ & $115^{\circ} 24^{\prime} 03^{\prime \prime}$ & 5-Aug-87 & -12.1 & -87 & -6.2 & 9.2 & 19720 \\
\hline Well, Amargosa Valley & $80 c .22, T 16 S$, R48E & $36^{\circ} 32^{\prime} 52^{\prime \prime}$ & $116^{\circ} 32^{\prime} 30^{\prime \prime}$ & 12-May-81 & -11.9 & -99 & -8.4 & 28.4 & 10405 \\
\hline Well, Amargosa Velley & 8ec. 16, T16S, R49E & $36^{\circ} 33^{\prime} 20^{\prime \prime}$ & $116^{\circ} 28^{\prime} 09^{\prime \prime}$ & 6-May-81 & -11.6 & -98 & -5.2 & 25.8 & 11200 \\
\hline Woll, SE side of Desert Range & sec.14, T16S, R58E & $36^{\circ} 33^{\prime} 32^{\prime \prime}$ & $116^{\circ} 24^{\prime} 40^{\prime \prime}$ & 4-Fob-89 & -12.6 & -92 & -7.7 & 1.3 & 35900 \\
\hline Indlan Springs & 80C.16, T16S, R56E & $36^{\circ} 33^{\prime} 54^{\prime \prime}$ & $115^{\circ} 40^{\prime} 06^{\prime \prime}$ & - & $-13.3(3)$ & $-99(3)$ & -7.8 & 7.7 & 21195 \\
\hline Well, Amargosa Valloy & sec.15, T16S, R48E & $36^{\circ} 34^{\prime} 05^{\prime \prime}$ & $116^{\circ} 32^{\prime} 40^{\prime \prime}$ & 6-May-81 & -13.0 & -103 & -7.1 & 18.0 & 14200 \\
\hline Well, Amargosa Valley & sec.07, T16S, R50E & $36^{\circ} 34^{\prime} 25^{\prime \prime}$ & $116^{\circ} 23^{\prime} 60^{\prime \prime}$ & 8-May-81 & -13.4 & -104 & -4.0 & 7.5 & 21410 \\
\hline Well, Amargosa Valley & Sec. 10, T16S, R48E & $36^{\circ} 34^{\prime} 25^{n}$ & $116^{\circ} 33^{\prime} 20^{n}$ & 10-May-81 & -12.2 & -101 & -5.0 & 16.5 & 14895 \\
\hline Indian Springs AFB Woll 1 & sec.08, T16S, R56E & $36^{\circ} 34^{\prime} 47^{n}$ & $115^{\circ} 40^{\prime} 47^{\prime \prime}$ & 27-Jun-85 & -13.0 & -96 & -6.9 & 7.3 & 21640 \\
\hline Amargosa Well \#5, Amarnosa Valley & sec. 08, T16S, R49E & $36^{\circ} 34^{\prime} 56^{\prime \prime}$ & $118^{\circ} 28^{\prime} 41^{\prime \prime}$ & 17-Nov-72 & -13.2 & -100 & -6.8 & 21.4 & 12745 \\
\hline Cow Camo Sprimg, Sheep Range & sec.02, T16S, R59E & $36^{\circ} 35^{\prime} 01^{\prime \prime}$ & $115^{\circ} 18^{\prime} 26^{\prime \prime}$ & - & $-12.6(2)$ & $-92(2)$ & & & \\
\hline Amargosa Well \#4, Amargosa Velley & 80C.05, T16S, R49E & $36^{\circ} 35^{\prime} 28^{\prime \prime}$ & $116^{\circ} 28^{\prime} 42^{\prime \prime}$ & 4-Mar-74 & -13.2 & -103 & -7.1 & 19.3 & 13600 \\
\hline Amy Well \#1, Meroury Valloy & Sec.05, T16S, R53E & $36^{\circ} 35^{\prime} 30^{\prime \prime}$ & $116^{\circ} 02^{\prime} 14^{\prime \prime}$ & - & $-13.4(3)$ & $-100(3)$ & $-8.2(3)$ & $4.9(4)$ & 24935 \\
\hline Well, Amargosa Valley 15S/49E-22dc & sec.22, T15S, R49E & $36^{\circ} 37^{\prime} 40^{\prime \prime}$ & $116^{\circ} 26^{\prime} 39^{\prime \prime}$ & - & -12.8 & -102 & & 15.6 & 15360 \\
\hline Wiregrass Spring, Shoep Range & sec.13, T158, R60E & $36^{\circ} 38^{\prime} 00^{n}$ & $115^{\circ} 12^{\prime} 29^{\prime \prime}$ & - & $-12.8(10)$ & $-94(10)$ & -10.2 & 96.8 & 289 \\
\hline Mormon Well, E. of Sheep Renge & Soc.12, T15S,R61E & $36^{\circ} 38^{\prime} 38^{\prime \prime}$ & $115^{\circ} 05^{\prime} 52^{\prime \prime}$ & - & $-12.7(3)$ & $-92(3)$ & & & \\
\hline
\end{tabular}


Table 5. Summary of lsotoplc Data complled by GeoTrans, Inc.

\begin{tabular}{|c|c|c|c|c|c|c|c|c|c|}
\hline Sample Name & Location & Latitude & Longltude & Date & $8^{18} \mathrm{O}$ & $8 D$ & $8^{13} \mathrm{C}$ & ${ }^{14} \mathrm{C}$ & ${ }^{14} \mathrm{C}$ app. age \\
\hline & & & & $i$ & $\left(\infty_{0}\right)$ & $\left(x_{0}\right)$ & $\left(\infty_{0}\right)$ & (pmc) & (years) \\
\hline Keene Wonder Springs, Death Valley, CA & sec.01, T15S, R46E & $36^{\circ} 40^{\prime} 25^{\prime \prime}$ & $11.66^{\circ} 55^{\prime} 11^{\prime \prime}$ & 23-Apr-82 & -13.0 & -100 & & & \\
\hline Sawmill Spring, Sheop Range & $80 C .30$, T14S, R81E & $36^{\circ} 40^{\prime} 50^{\prime \prime}$ & $115^{\circ} 10^{\prime} 34^{\prime \prime}$ & $19-$ May -88 & -12.9 & -92 & & & \\
\hline Whilte Rock Spring, Sheep Range & $\mathrm{SOC.} 22, \mathrm{~T} 14 \mathrm{~S}, \mathrm{RBOE}$ & $36^{\circ} 42^{\prime} 30^{n}$ & $115^{\circ} 14^{\prime} 20^{\prime \prime}$ & - & $-9.9(2)$ & $-84(2)$ & & & \\
\hline J-12 well, Area 25, Nevada Test Site & $80 C .06, T 14 S, R 50 E$ & $36^{\circ} 45^{\prime} 54^{\prime \prime}$ & $116^{\circ} 23^{\prime} 24^{\prime \prime}$ & - & $-13.1(5)$ & $-97(6)$ & & 30.5 (2) & 9820 \\
\hline Well, U.S. Ecology Site, Amargosa Desert & $80 C .35, T 13 S$, R47E & $36^{\circ} 45^{\prime} 66^{\prime \prime}$ & $116^{\circ} 41^{\prime} 35^{\prime \prime}$ & $16-$ Aug-89 & -14.0 & -108 & -6.7 & 26.2 & 11070 \\
\hline Well, U.S. Ecology Site, Amargosa Desert & sec.35, T13S, R47E & $36^{\circ} 45^{\prime} 57^{\prime \prime}$ & $116^{\circ} 41^{\prime} 14^{\prime \prime}$ & 9-Dec-92 & -13.8 & -104 & -6.5 & 17.1 & 14190 \\
\hline Well, U.S. Ecology Stite, Amargosa Desert & $800.35, T 13 S$, R47E & $36^{\circ} 45^{\prime} 57^{\prime \prime}$ & $116^{\circ} 41^{\prime} 15^{\prime \prime}$ & 9-Dec-92 & -13.9 & -104 & -7.8 & 13.8 & 16370 \\
\hline Well, U.S. Ecology Site, Amargosa Desert & sec, 35, T13S, R47E & $36^{\circ} 46^{\prime} 01^{\prime \prime}$ & $116^{\circ} 41^{\prime} 41^{\prime \prime}$ & 8-Dec-92 & -14.0 & -104 & -6.7 & 25.0 & 11460 \\
\hline Well, U.S. Ecology Slite, Amargosa Desert & BOC.26, T13S, R47E & $36^{\circ} 46^{\prime} 15^{\prime \prime}$ & $116^{\circ} 411^{\prime 2} 4^{\prime \prime}$ & - & $-14.0(2)$ & $-106(2)$ & $-7.2(2)$ & $14.6(2)$ & 15935 \\
\hline USW VH-1 well, Grater Flat & $80 C, 27, T 13 S, R 48 E$ & $36^{\circ} 47^{\prime} 32^{n}$ & $116^{\circ} 33^{\prime} 07^{\prime \prime}$ & $=$ & -14.2 & -108 & -8.5 & 12.2 & 17390 \\
\hline J-13 well, Area 26, Nevada Test Slte & $80 C .19$, T13S, R5OE & $36^{\circ} 48^{\prime} 28^{\prime \prime}$ & $116 \cdot 23^{\prime} 40^{n}$ & - & $-13.2(3)$ & $-96(3)$ & .7 .3 & $29.9(2)$ & 9990 \\
\hline UE-25p-1 well, Yuoca Mitn. & sec.14, T13S, R49E & $36^{\circ} 49^{\prime} 38^{\prime \prime}$ & $116^{\circ} 25^{\prime} 21^{\prime \prime}$ & - & $-13.8(2)$ & $-107(2)$ & & & \\
\hline UE-25o-2 woll, Yucca Mitn & $800.14, T 13 S, \mathrm{R} 49 \mathrm{E}$ & $36^{\circ} 49^{\prime} 45^{\prime \prime}$ & $116^{\circ} 25^{\prime} 43^{\prime \prime}$ & 13-Mar-84 & -13.4 & -101 & -7.0 & 15.3 & 15520 \\
\hline UE-25c-3 woll, Yucca Minn & SeC. 14, T13S, R49E & $36^{\circ} 49^{\prime} 47^{\prime \prime}$ & $116^{\circ} 25^{\prime} 43^{\prime \prime}$ & - & $-13.5(2)$ & $-103(2)$ & $-7.3(2)$ & $15.4(2)$ & 15490 \\
\hline USW H-4 well, Yuoca Min. & $800.36, T 12 S, R 49 E$ & $38^{\circ} 50^{\prime} 32^{\prime \prime}$ & $116^{\circ} 26^{\prime} 54^{\prime \prime}$ & 17-May-82 & -14.0 & -104 & -7.4 & 11.8 & 17670 \\
\hline USW H-6 well, Yucca Mtn. & sec.34, T12S, R49E & $36^{\circ} 50^{\prime} 49^{\prime \prime}$ & $116^{\circ} 28^{\prime} 55^{\prime \prime}$ & $16-0 c t-82$ & -13.8 & -106 & -7.5 & 17.1 & 14600 \\
\hline Tim Spring, Pintwater Raxge & $800.05, T 13 S$, R57E & $38^{\circ} 50^{\prime} 58^{\prime \prime}$ & $115^{\circ} 34^{\prime} 10^{n}$ & $2-\sqrt{a n}-88$ & -13.2 & -99 & & & \\
\hline UE-25b-1 well, Yucca Mith. & sec.31, T12S, R50E & $38.51108 "$ & $116^{\circ} 26^{\prime} 23^{\prime \prime}$ & - & $-13.2(3)$ & $-100(3)$ & $-9.5(2)$ & 18.2 (2) & 14080 \\
\hline USW G-4 well, Yucca Mith. & $800.36, T 12 S, R 49 E$ & $36^{\circ} 51^{\prime} 14^{\prime \prime}$ & $116^{\circ} 27^{\prime} 04^{\prime \prime}$ & 9-Dec-82 & -13.8 & -103 & -9.1 & 22.0 & 12520 \\
\hline Well, 5 miles SW of Beatty & sec.27, T12S, R46E & $36^{\circ} 51^{\prime} 28^{\prime \prime}$ & $116^{\circ} 49^{\prime} 20^{\prime \prime}$ & $9-J u l-88$ & -13.5 & -102 & & & \\
\hline USW H-1 Piezometer 4, Yucea Min. & $80 C .25, T 12 S, R 49 E$ & $36^{\circ} 51^{\prime} 57^{n}$ & $116 \cdot 27^{\prime} 12^{n}$ & 8-Dec-82 & -13.5 & -101 & -11.4 & 23.9 & 11830 \\
\hline Well nr. Amargosa R. 12s/47E-19abc & $80 C .19, T 12 S$, R47E & $36^{\circ} 52^{\prime} 45^{\prime \prime}$ & $118^{\circ} 45^{\prime} 18^{\prime \prime}$ & - & -13.3 & -104 & -8.0 & 92.8 & 614 \\
\hline Spring, S. of Bealty 128/47E-20bbb & soc.20, T12S, R47E & $36^{\circ} 53^{\prime} 08^{\prime \prime}$ & $116^{\circ} 45^{\prime} 00^{n}$ & $=$ & -13.6 & -106 & -8.0 & & \\
\hline Sheep Spring, Shoep Range & SOC.23, T12S, R61E & $36^{\circ} 53^{\prime} 42^{n}$ & $115^{\circ} 00^{\prime} 63^{\prime \prime}$ & $\overline{-}$ & $-13.6(2)$ & $-102(2)$ & -7.0 & 64.7 & 4000 \\
\hline Well, Beatty 12S/47E-0oodd & sec.06, T12S, R47E & $36^{\circ} 54^{\prime} 55^{\prime \prime}$ & $116^{\circ} 45^{\prime} 40^{n}$ & $=$ & -13.3 & -102 & $-6.9(2)$ & $37.0(2)$ & 8230 \\
\hline Beatty Spring, N. side of Beatty Mtn. & $80 C .05, T 12 S$, R47E & $38^{\circ} 55^{\prime} 07^{\prime \prime}$ & $116^{\circ} 44^{\prime} 37^{\prime \prime}$ & $=$ & -14.1 & -108 & $-7.0(2)$ & $34.2(2)$ & 8880 \\
\hline UE-29a-2 well, Nevada Test Site & $300.34, T 11 S, R 50 E$ & $36^{\circ} 56^{\prime} 29^{\prime \prime}$ & $116^{\circ} 22^{\prime} 26^{\prime \prime}$ & - & -12.8 & -93 & $-12.8(3)$ & $66.0(3)$ & 3435 \\
\hline Lamb Spring, Sheop Range & $806.35, T 11 S, R 61 E$ & $36^{\circ} 56^{\prime} 42^{n}$ & $115^{\circ} 06^{\prime} 21^{\prime \prime}$ & 19-Мау-88 & -13.2 & -93 & & & \\
\hline Spring, Oasls Valloy 11S/47E-28dac & Sec.28, T11S, R47E & $36^{\circ} 57^{\prime} 00^{\prime \prime}$ & $116^{\circ} 43^{\prime} 00^{\prime \prime}$ & - & -14.1 & -109 & -6.9 & & \\
\hline Desert Lake Well, Desert Valley & $800.36, T 11 S, R 60 E$ & $36 \cdot 57^{\prime} 11^{\prime \prime}$ & $115^{\circ} 11^{\prime} 51^{\prime \prime}$ & 18-Mar-87 & -13.1 & .98 & -5.3 & 1.3 & 36900 \\
\hline Well, Oasls Valley 11S/47E-28aac & sec. $28, T 11 S, R 47 E$ & $36^{\circ} 57^{\prime} 18^{\prime \prime}$ & $116^{\circ} 43^{\prime} 10^{\prime \prime}$ & - & -14.1 & -108 & -7.1 & & \\
\hline Spring, Osals Valloy 118/47E-21dbb & $80 c .21, T 11 S, R 47 E$ & $36^{\circ} 57^{\prime} 56^{\prime \prime}$ & $116^{\circ} 43^{\prime} 20^{\prime \prime}$ & - & -14.0 & -109 & -4.5 & & \\
\hline Plapi Canyon, Shoshone Mth., NTS & sec.14, T11S, R51E & $36^{\circ} 58^{\prime} 30^{\prime \prime}$ & $116^{\circ} 15^{\prime} 29^{\prime \prime}$ & $11-F e b-81$ & .14 .2 & -108 & -8.5 & 12.8 & 17000 \\
\hline Quartz Spring, PIntwater Range & Sec.17, T11S, R57E & $36^{\circ} \cdot 59^{\prime} 10^{n}$ & $115^{\circ} 36^{\prime} 00^{\prime \prime}$ & $2-\operatorname{Jan}-88$ & -11.6 & -88 & & & \\
\hline Surprise Springs, Death Valley, CA & Soc. 18, T11S, R43E & $37^{\circ} 00^{\prime} 08^{n}$ & $117^{\circ} 20^{\prime} 37^{\prime \prime}$ & 20-App-82 & -14.5 & -110 & -6.9 & $23.0(2)$ & 12165 \\
\hline
\end{tabular}


Table 5. Summary of leotoplc Data complied by GeoTrans, Inc.

\begin{tabular}{|c|c|c|c|c|c|c|c|c|c|}
\hline Sample Name & Location & Latitude & Longitude & Dete & $8^{18} \mathrm{O}$ & $8 \mathrm{D}$ & $8^{13} \mathrm{C}$ & ${ }^{14} \mathrm{C}$ & ${ }^{14} \mathrm{C}$ spp. age \\
\hline & & & & 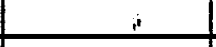 & $\left(\psi_{0}\right)$ & (\$o) & $(\%)$ & (pmc) & (years) \\
\hline Spring, Oasis Valley 118/47E-04cad & 8e0.04, T11S, R47E & $37^{\circ} 00^{\prime} 30^{n}$ & $116^{\circ} 43^{\prime} 30^{\prime \prime}$ & - & -14.0 & -108 & -5.0 & & \\
\hline Spring, Oasis Valloy 10s/47E-32dda & sec.32, T10S, R47E & $37^{\circ} 01^{\prime} 11^{\prime \prime}$ & $116^{\circ} 44^{\prime} 00^{\prime \prime}$ & - & -13.4 & -102 & -6.0 & & \\
\hline Grapevine Springs, Death Valley, CA & sec.10, T118, R42E & $37^{\circ} 01^{\prime} 11^{\prime \prime}$ & $117^{\circ} 23^{\prime} 11^{\prime \prime}$ & 19-Apr-82 & -14.2 & -109 & & & \\
\hline Spring, Oasis Valloy 108/47E-33aab & sec. 33, T10s, R47E & $37^{\circ} 01^{\prime} 49^{\prime \prime}$ & $116^{\circ} 43^{\prime} 07^{\prime \prime}$ & - & -14.0 & -108 & -4.9 & 100.0 & modern \\
\hline Peacock Spring, Springdale, Oasls Valley & sec.31, T10S, R47E & $37^{\circ} 01^{\prime} 51^{\prime \prime}$ & $116^{\circ} 45^{\prime} 17^{\prime \prime}$ & - & -13.4 & -102 & -5.3 & 99.8 & 18 \\
\hline Springs, $1 \mathrm{ml}$. E of Scotty's Castle, CA & $80 c .05, T 11 \mathrm{~S}, \mathrm{R} 43 \mathrm{E}$ & $37^{\circ} 01^{\prime} 56^{\prime \prime}$ & $117^{\circ} 19^{\prime} 29^{\prime \prime}$ & 20-Apr-82 & -14.5 & -110 & -5.2 & $19.6(2)$ & 13490 \\
\hline Well, Oasis Valley 10S/47E-27cba & sec.27, T10S, R47E & $37^{\circ} 02^{\prime} 16^{\prime \prime}$ & $116^{\circ} 42^{\prime} 42^{\prime \prime}$ & - & -14.3 & -111 & -5.3 & 38.9 & 7805 \\
\hline Well, Oasis Valley 10S/47E-30acc & Sec. 30, T10S, R47E & $37^{\circ} 02^{\prime} 18^{\prime \prime}$ & $116^{\circ} 45^{\prime} 20^{\prime \prime}$ & $=$ & -13.4 & -102 & -5.2 & 35.0 & 8680 \\
\hline Spring, Oasis Valley 10s/47E-14bab & sec. 14, T10S, R47E & $37^{\circ} 04^{\prime} 27^{\prime \prime}$ & $116^{\circ} 41^{\prime} 24^{\prime \prime}$ & - & -14.5 & -112 & $-4.0(2)$ & $10.7(2)$ & 18475 \\
\hline Maynard Lake Spring, Pahranegat Valley & SeC.04, T9S, R62E & $37^{\bullet} 11^{\prime} 30^{n}$ & $115^{\circ} 02^{\prime} 02^{\prime \prime}$ & 14-Jan-85 & -12.3 & -94 & & & \\
\hline Lone Tree Spring, Pahranegat Valley & sec.05, T9S, R62E & $37^{\circ} 12^{\prime} 07^{\prime \prime}$ & $15^{\circ} 03^{\prime} 32^{n}$ & 14-Jan-85 & -10.9 & -90 & & & \\
\hline U-20a 2 well, Nevada Test Site & sec.17, T8S, R50E & $37^{\circ} 14^{\prime} 34^{\prime \prime}$ & $116^{\circ} 26^{\prime} 16^{\prime \prime}$ & $=$ & -14.8 & -114 & -13.5 & 15.3 & 15520 \\
\hline UE-19gs mell, Nevada Test Slte & 8ec.26, T7S; RSOE & $37^{\circ} 18^{\prime} 30^{\prime \prime}$ & $116^{\circ} 21^{\prime} 53^{\prime \prime}$ & - & -14.5 & -114 & & & \\
\hline Cane Spring, Groom Range & sec. 09, T7S, R55.5E & $37^{\circ} 20^{\prime} 27^{\prime \prime}$ & $115^{\circ} 44^{\prime} 60^{\prime \prime}$ & 15-May-85 & -13.2 & -92 & & & \\
\hline Cattle Spring, Croom Ranoe & 800.16, T6S, R55.5E & $37^{\circ} 24^{\prime} 67^{\prime \prime}$ & $115^{\circ} 45^{\prime} 05^{\prime \prime}$ & 15-May-85 & -9.4 & -85 & & & \\
\hline Rock Spring, Croom Range & $800.16, T 68$, R56E & $37^{\circ} 25^{\prime} 53^{\prime \prime}$ & $115^{\circ} 41^{\prime} 23^{\prime \prime}$ & 15-May-85 & -10.8 & -86 & & & \\
\hline Quall Spring, Groom Range & Sec.09, T6S, R56E & $37^{\circ} 26^{\prime} 29^{\prime \prime}$ & $115^{\circ} 41^{\prime} 01^{\prime \prime}$ & 15-May-85 & -11.9 & -92 & & & \\
\hline Hells Acre Gukch Soring, Hilko Range & sec. $03, T 65$, R61E & $37^{\circ} 27^{\prime} 37^{\prime \prime}$ & $11^{\circ} 07^{\prime} 29^{\prime \prime}$ & 14-Jan-85 & -12.3 & -93 & & & \\
\hline Ash Sorings, Pahranagat Valloy & 860,06, T6S, R61E & $37^{\circ} 27^{\prime} 49^{\prime \prime}$ & $116^{\circ} 11^{\prime} 30^{\prime \prime}$ & 20-Jul-81 & -14.1 & -108 & & 6.8 & 22220 \\
\hline Indian Spring, Groom Ranoe & $80 c .33$, T5S, R55.6E & $37^{\circ} 27^{\prime} 50^{\prime \prime}$ & $115^{\circ} 44^{\prime} 28^{\prime \prime}$ & 15-May-85 & -10.4 & -88 & & & \\
\hline Rabbltbrush Sping, Groom Range & 80c.28, T5S, R56E & $37^{\circ} 28^{\prime} 56^{\prime \prime}$ & $15^{\circ} 41^{\prime} 28^{\prime \prime}$ & 14-May-85 & -11.2 & -89 & & & \\
\hline Bullwhack Spring, Groom Range & sec.20, T5S, R55.5E & $37^{\circ} 29^{\prime} 45^{\prime \prime}$ & $115^{\circ} 45^{\prime} 47^{\prime \prime}$ & 17-May-85 & -10.2 & -88 & & & \\
\hline Crystal Springs, Pahranagat Valley & sec. 10, T5S, R6OE & $37^{\circ} 31^{\prime} 53^{\prime \prime}$ & $115^{\circ} 13^{\prime} 58^{\prime \prime}$ & 20-Jul-81 & -14.3 & -109 & & 7.8 & 21090 \\
\hline April Fool Spring. Groom Range & sec. 04, T5S, R55.5E & $37^{\circ} 32^{\prime} 14^{\prime \prime}$ & $115^{\circ} 44^{\prime} 18^{\prime \prime}$ & 16-May-85 & -10.7 & -89 & & & \\
\hline Hiko Spring, Pahranagat Valley & sec.14, T4S, R60E & $37^{\circ} 35^{\prime} 54^{\prime \prime}$ & $115^{\circ} 12^{\prime} 52^{\prime \prime}$ & 14-Jan-85 & -15.3 & -110 & -7.0 & 6.5 & 22600 \\
\hline Sand Spring, Sand Spring Valley & 800.26, T2S, R56E & $37^{\circ} 44^{\prime} 20^{\prime \prime}$ & $115^{\circ} 45^{\prime} 06^{\prime \prime}$ & $3-$ Jan-88 & -11.9 & -88 & & & \\
\hline The Seeps, Timpahute Range & sec.28, T2S, R57E & $37^{\circ} 44^{\prime} 22^{\prime \prime}$ & $115^{\circ} 34^{\prime} 32^{\prime \prime}$ & 15-Jan-85 & -13.3 & -98 & & & \\
\hline Cedar Pass Well, Cactus Flat & 8ec.22, T2S, R49E & $37^{\circ} 44^{\prime} 48^{\prime \prime}$ & $116^{\circ} 28^{\prime} 58^{\prime \prime}$ & 10-Sep-80 & -14.0 & -110 & & & \\
\hline Cedar Spring, Kawich Range & Sec.22, T2S, R51E & $37^{\circ} 45^{\prime} 05^{\prime \prime}$ & $116^{\circ} 16^{\prime} 25^{\prime \prime}$ & 15-Jan-85 & -13.6 & -101 & & & \\
\hline Well 9, Cactus Flat, Toriopah Test Range & 80c.16, T1S, R47E & $37^{\circ} 51^{\prime} 15^{\prime \prime}$ & $116^{\circ} 43^{\prime} 06^{\prime \prime}$ & - & -14.3 & -113 & & & \\
\hline Georges Water, Kawich Range & 8ec.14, T1S; R50E & $37^{\circ} 51^{\prime} 37^{\prime \prime}$ & $116^{\circ} 20^{\prime} 57^{\prime \prime}$ & 15-Jan-85 & -13.6 & -98 & & & \\
\hline Well 1A, Cactus Flat, Tonopah Test Range & $806,01, T 15$, R46E & $37^{\circ} 53^{\prime} 00^{\prime \prime}$ & $116^{\circ} 46^{\prime} 53^{\prime \prime}$ & - & -14.5 & -118 & & & \\
\hline Eden Creek Ranch Spring, Kawich Range & Sec.04, TIN, R50E & $37^{\bullet} 58^{\prime} 12^{n}$ & $116^{\circ} 22^{\prime} 53^{\prime \prime}$ & 15-Jan-85 & -13.9 & -99 & & & \\
\hline Unnamed Spring, Colden Cate Range & $80 C .09$, T3N, R59E & $38^{\circ} 07^{\prime} 58^{\prime \prime}$ & $115^{\circ} 20^{\prime} 46^{\prime \prime}$ & 15-Jan-81 & -14.6 & -110 & & 10.6 & 18650 \\
\hline Adavan Spring, Quinn Camon Range & 806.08, T3N, R57E & $38^{\circ} 08^{\prime} 05^{\prime \prime}$ & $115^{\bullet} 35^{\prime} 68^{\prime \prime}$ & $31-\mathrm{Jul}-85$ & -14.0 & -103 & & & \\
\hline
\end{tabular}


Table 5. Summary of lsotople Data compiled by GeoTrans, Inc.

\begin{tabular}{|c|c|c|c|c|c|c|c|c|c|}
\hline Sample Name & Location & Latitude & Longitude & Date & $\delta^{18} 0$ & 80 & $8^{13} \mathrm{C}$ & ${ }^{14} \mathrm{C}$ & ${ }^{14} \mathrm{C}$ app. age \\
\hline & & & & $=$ & $\left(\infty_{0}\right)$ & $\left(x_{0}\right)$ & $\left(\infty_{0}\right)$ & (pmc) & (years) \\
\hline Little Cherry Creek, Quinn Canyon Range & 800.35, T 4N, R56E & $38^{\circ} 10^{\prime} 02^{\prime \prime}$ & $115^{\circ} 39^{\prime} 12^{n}$ & $31-J u l-85$ & -13.9 & -103 & & & \\
\hline Warm Springs, Jet. Hwy 6 and 375 & $80 c .20, T 4 N, R 50 E$ & $38^{\circ} 11^{\prime} 15^{\prime \prime}$ & $116^{\circ} 22^{\prime} 21^{n}$ & $1-$ Jan-74 & -14.2 & -114 & & & \\
\hline Moon River Spring, Whilte Rlver Valley & SeC.25, T6N, R6OE & $38 \cdot 21^{\prime} 08^{\prime \prime}$ & $115 \cdot 10^{\prime} 50^{\prime \prime}$ & $27-A p r-82$ & -15.8 & -120 & -7.5 & 6.4 & 22720 \\
\hline Hot Creek Spring, White Rlver Valloy & SEC.18, T6N, R61E & $38^{\circ} 22^{\prime} 59^{\prime \prime}$ & $115^{\circ} 09^{\prime} 09^{\prime \prime}$ & 19-Jul-81 & -15.5 & -118 & & 4.6 & 25510 \\
\hline Hot Creek discharge area & Se0.09, T6N, R61E & $38^{\circ} 23^{\prime} 18^{\prime \prime}$ & $115^{\circ} 07^{\prime} 58^{\prime \prime}$ & $19-J u l-81$ & -15.3 & -118 & & & \\
\hline Flag Spring \#3, White River Valley & $80 C .33, T 7 N, R 62 E$ & $380^{\circ} 25^{\prime} 17^{\prime \prime}$ & $115^{\circ} 01^{\prime} 20^{\prime \prime}$ & 17-Jan-84 & -14.3 & -105 & & & \\
\hline Bullwhacker Springs, Railroad Valloy & Sec. $28, T 7 N, R 57 E$ & $38^{\circ} 26^{\prime} 20^{\prime \prime}$ & $115^{\circ} 34^{\prime} 08^{\prime \prime}$ & 16-Jun-83 & -15.2 & -114 & & & \\
\hline Butterfield Spr, Whitso Aiver Valloy & $800.28, T 7 N, R 62 E$ & $38^{\circ} 26^{\prime} 23^{\prime \prime}$ & $115^{\circ} 00^{\prime} 39^{\prime \prime}$ & $10-\mathrm{Jul}-81$ & -14.2 & -105 & & 31.3 & 9600 \\
\hline Butterfield Sprs, Rallroad Valloy & SOC.27, T8N, R57E & $38^{\circ} 31^{\prime} 16^{n}$ & $115^{\circ} 32^{\prime} 46^{\prime \prime}$ & 15-Jun-83 & -15.0 & -116 & & & \\
\hline Big Spring, Rallroad Valley & Sec. 15, T8N, R55E & $38^{\circ} 33^{\prime} 21^{\prime \prime}$ & $116^{\circ} 46^{\prime} 15^{\prime \prime}$ & 16-Jul-81 & -15.4 & -120 & & 3.0 & 28990 \\
\hline Blue Eagle Spring, Rallroad Valley & Sec.11, T8N, R57E & $38^{\circ} 33^{\prime} 47^{\prime \prime}$ & $115^{\circ} 31^{\prime} 39^{n}$ & 17-Jul-81 & -15.0 & -114 & & 10.3 & 18800 \\
\hline Albert Spring, Grant Range & SeC.08, T8N, R59E & $38^{\circ} 34^{\prime} 08^{\prime \prime}$ & $115^{\circ} 21^{\prime} 42^{\prime \prime}$ & 24-Jul-85 & -14.0 & -107 & & & \\
\hline Moorman Spring, White River Valley & 800.32, T9N, R61E & $38^{\circ} 35^{\prime} 41^{\prime \prime}$ & $115^{\circ} 08^{\prime} 18^{\prime \prime}$ & 18-Jul-81 & -15.7 & -119 & & 4.2 & 26210 \\
\hline Emigrent Springe, White River Valley & 8ec.19, T9N, R62E & $38^{\circ} 37^{\prime} 26^{\prime \prime}$ & $115^{\circ} 02^{\prime} 61^{\prime \prime}$ & $18-\mathrm{JuI}-81$ & -14.5 & -108 & & 56.6 & 4705 \\
\hline Rogers Ranch Springs, Blg Smoky Valley & sec.20, T11N, R43E & $38^{\circ} 48^{\prime} 15^{\prime \prime}$ & $117^{\circ} 10^{\prime} 48^{\prime \prime}$ & $1-\operatorname{Jan}-74$ & -15.5 & -123 & & & \\
\hline Darrough Hot Springe, Blo Smoky Valloy & sec.18, T11N, R43E & $38^{\circ} 49^{\prime} 09^{\prime \prime}$ & $117^{\circ} 10^{\prime} 58^{\prime \prime}$ & $1-\operatorname{Jan}-74$ & -15.3 & -119 & & & \\
\hline Secret Spring, Horee Range & sec.01, T11N, R59E & $38^{\circ} 50^{\prime} 20^{\prime \prime}$ & $115^{\circ} 17^{\prime} 23^{n}$ & $16-\sqrt{u n}-83$ & -14.0 & -110 & & & \\
\hline Lund Spring, White River Valley & BeC.04, T11N, R62E & $38^{\circ} 51^{\prime} 00^{\prime \prime}$ & $115^{\circ} 00^{\prime} 09^{\prime \prime}$ & $27-A p r-82$ & -15.4 & -113 & & & \\
\hline Little Currant Creek Sprs. & $80 c .18, T 12 N, R 59 E$ & $38^{\circ} 53^{\prime} 39^{n}$ & $115^{\cdot} \cdot 22^{\prime} 58^{n}$ & 15-Jun-83 & -14.0 & -107 & & & \\
\hline Little Currant Creek Sprs. & SeC. $18, T 12 \mathrm{~N}, \mathrm{R} 59 \mathrm{E}$ & $38^{\circ} 54^{\prime} 02^{\prime \prime}$ & $15^{\circ} 22^{\prime} 57^{\prime \prime}$ & 15-Jun-83 & -14.1 & -105 & & & \\
\hline Unnamed Spring, White Plne Range & sec.07, T12N, R59E & $38^{\circ} 54^{\prime} 36^{\prime \prime}$ & $115^{\circ} 23^{\prime} 11^{\prime \prime}$ & $15-\mathrm{Jun}-83$ & -15.0 & -116 & & & \\
\hline Nicholas Springs, White River Valley & $80 C, 12, T 12 N, R 61 E$ & $38^{\circ} 54^{\prime} 45^{\prime \prime}$ & $115^{\circ} 03^{\prime} 37^{\prime \prime}$ & $27-A p r-82$ & -16.1 & -124 & -8.0 & 6.5 & 22600 \\
\hline Indian Ranch Spring, White River Valley & sec.02, T12N, R61E & $38^{\circ} 55^{\prime} 38^{\prime \prime}$ & $115^{\circ} 04^{\prime} 57^{\prime \prime}$ & - & $-15.8(2)$ & $-124(2)$ & -5.9 & $2.3(2)$ & 31110 \\
\hline Preston Big Spring, White River Valley & sec.02, T12N, R61E & $38^{\circ} 56^{\prime} 01^{n}$ & $115^{\circ} 04^{\prime} 50^{n}$ & - & $-15.8(2)$ & $-125(2)$ & $-5.8(2)$ & $12.5(2)$ & 17190 \\
\hline Well, $4 \mathrm{~km}$ ESE of Gandner Ranch & sec.33, T13N, R60E & $38^{\circ} 56^{\prime} 52^{\prime \prime}$ & $115^{\circ} 13^{\prime} 41^{\prime \prime}$ & 29-Apr-82 & -15.8 & -118 & & & \\
\hline Big Warm Spring, Duckwater & Sec.32, T13N, R56E & $38 \cdot 57^{\prime} 01^{n}$ & $115^{\circ} 42^{\prime} 08^{\prime \prime}$ & 13-Jun-83 & -15.9 & -125 & -4.4 & 3.1 & 28720 \\
\hline Diana's Punch Bowl (Hot Spr) & $80 c .22, T 14 N, R 47 E$ & $39^{\circ} 01^{\prime} 47^{\prime \prime}$ & $116^{\circ} 40^{\prime} 02^{\prime \prime}$ & $1-\operatorname{Jan}-74$ & -16.2 & -125 & & & \\
\hline Butler Ranch, Monitor Valloy & $80 c .02, T 14 N, R 48 E$ & $39^{\circ} 04^{\prime} 57^{\prime \prime}$ & $116^{\circ} 32^{\prime} 34^{\prime \prime}$ & $1-\operatorname{Jan}-74$ & -16.3 & -128 & & & \\
\hline Fish Creek Springs, Llttle Smoky Valley & sec.08, T16N, R53E & $39 \cdot 16^{\prime} 37^{\prime \prime}$ & $116^{\circ} 02^{\prime} 18^{\prime \prime}$ & 17-Jul-81 & -15.6 & -121 & & 3.1 & 28720 \\
\hline USGS Well \#23, Smlth CK. Valley & $80 C .04, T 16 \mathrm{~N}, \mathrm{R} 40 \mathrm{E}$ & $39^{\circ} 16^{\prime} 69^{\prime \prime}$ & $117^{\circ} 29^{\prime} 02^{\prime \prime}$ & 5-Aug-82 & -16.4 & -132 & & & \\
\hline USGS Well \#55, Smith CK. Valley & sec.27, T17N, R40E & $30^{\circ} 17^{\prime} 54^{\prime \prime}$ & $117^{\circ} 29^{\prime} 47^{\prime \prime}$ & 12-Aug-82 & .15 .2 & -124 & & & \\
\hline Spencer Hot Springs, Blg Smoky Valley & sec.13, T17N, R45.5E & $39^{\circ} 18^{\prime} 46^{\prime \prime}$ & $116^{\circ} 51^{1} 05^{\prime \prime}$ & 1-Jan-74 & -16.0 & -136 & & & \\
\hline Spencer Hot Springs, Blg Smoky Valley & $80 \mathrm{c} .12, \mathrm{~T} 17 \mathrm{~N}, \mathrm{R} 45.5 \mathrm{E}$ & $39^{\circ} 19^{\prime} 31^{\prime \prime}$ & $116^{\circ} 51^{\prime} 03^{\prime \prime}$ & 16-Jan-85 & -16.4 & -135 & & & \\
\hline Sand Spring, White Pine Range & $80 C .21, T 17 N, R 58 E$ & $39 \cdot 19^{\prime} 50^{n}$ & $116^{\circ} 27^{\prime} 18^{\prime \prime}$ & 14-Jul-81 & -16.2 & -123 & & & \\
\hline Wild Horse Spring, Whitte Pine Range & sec.21, T17N, R58E & $38^{\circ} 20^{\prime} 01^{\prime \prime}$ & $115^{\circ} 28^{\prime} 38^{n}$ & 14-Jul-81 & -16.8 & -129 & & & \\
\hline
\end{tabular}


Table 5. Summary of lsotopic Data complled by GeoTrans, Inc.

\begin{tabular}{|c|c|c|c|c|c|c|c|c|c|}
\hline Sample Name & Location & Latitude & Longltude & Date & $8^{18} \mathrm{O}$ & $8 D$ & $8^{13} \mathrm{C}$ & ${ }^{14} \mathrm{C}$ & ${ }^{14} \mathrm{C}$ app. age \\
\hline & & & & & $\left(\infty_{0}\right)$ & $\left(\infty_{0}\right)$ & $\left(\infty_{0}\right)$ & (pmc) & (years) \\
\hline Clobe Hot Spring, Antelope Valloy & $800.28, T 18 N$, R5OE & $39^{\circ} 24^{\prime} 10^{\prime \prime}$ & $116^{\circ} 20^{\prime} 24^{\prime \prime}$ & 1-Jan-74 & -16.3 & -128 & & & \\
\hline Blackbird Ranch Soring, Tolyabo Range & $80 c .18, T 18 N, R 45 E$ & $39^{\circ} 25^{\prime} 41^{\prime \prime}$ & $116^{\circ} 58^{\prime} 01^{\prime \prime}$ & 18 -Jan-86 & -15.4 & -119 & & & \\
\hline Blackbird Spring. Tolyabe Range & $800.01, \mathrm{~T} 18 \mathrm{~N}, \mathrm{R} 44 \mathrm{E}$ & $39^{\circ} 26^{\prime} 49^{\prime \prime}$ & $116^{\circ} 59^{\prime} 12^{n}$ & $13-J u l-81$ & -18.1 & -125 & & & \\
\hline
\end{tabular}




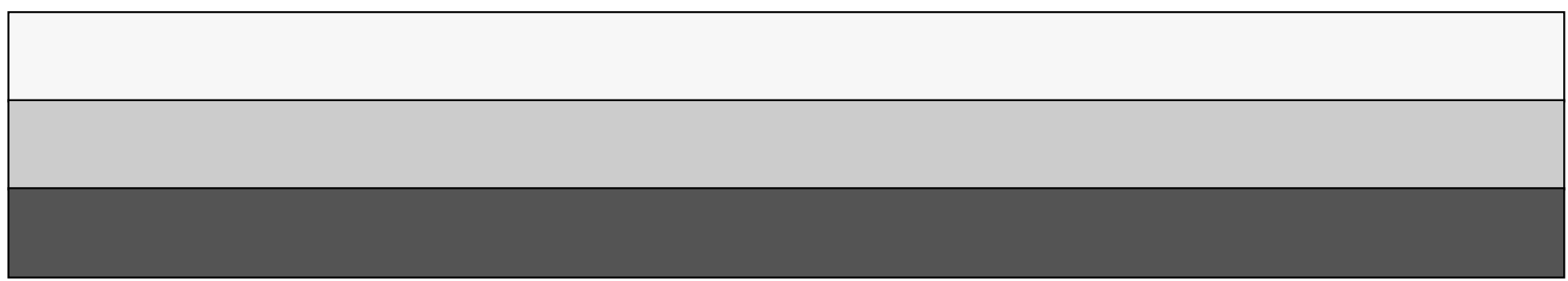

